\title{
A consistent two-factor model for pricing temperature derivatives
}

\author{
Andreas Groll* \\ Brenda López-Cabrera** \\ Thilo Meyer-Brandis*
}

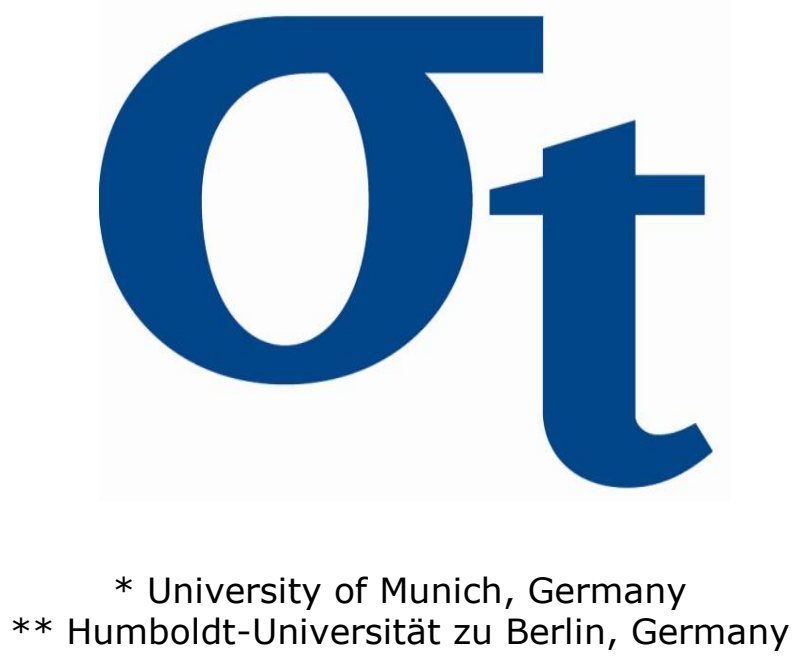

$\underline{Y}$

$\mapsto$

$\propto$

This research was supported by the Deutsche Forschungsgemeinschaft through the SFB 649 "Economic Risk".

http://sfb649.wiwi.hu-berlin.de ISSN 1860-5664 


\title{
A consistent two-factor model for pricing temperature
}

\author{
derivatives*
}

\author{
Andreas Groll ${ }^{\dagger} \quad$ Brenda López-Cabrera ${ }^{\ddagger} \quad$ Thilo Meyer-Brandis ${ }^{\S}$
}

\begin{abstract}
We analyze a consistent two-factor model for pricing temperature derivatives that incorporates the forward looking information available in the market by specifying a model for the dynamics of the complete meteorological forecast curve. The two-factor model is a generalization of the Nelson-Siegel curve model by allowing factors with mean-reversion to a stochastic mean for structural changes and seasonality for periodic patterns. Based on the outcomes of a statistical analysis of forecast data we conclude that the two-factor model captures well the stylized features of temperature forecast curves. In particular, a functional principal component analysis reveals that the model reflects reasonably well the dynamical structure of forecast curves by decomposing their shapes into a tilting and a bending factor. We continue by developing an estimation procedure for the model, before we derive explicit prices for temperature derivatives and calibrate the market price of risk (MPR) from temperature futures derivatives (CAT, HDD, CDD) traded at the Chicago Mercantile Exchange (CME). The factor model shows that the behavior of the implied MPR for futures traded in and out of the measurement period is more stable than other estimates obtained in the literature. This confirms that at least parts of the irregularity of the MPR is not due to irregular risk perception but rather due to
\end{abstract}

*The financial support from the Deutsche Forschungsgemeinschaft via SFB 649 "Ökonomisches Risiko", Humboldt-Universität zu Berlin is gratefully acknowledged.

${ }^{\dagger}$ Mathematical Institute, University of Munich, Theresienstrasse 39, 80333 Munich, Germany. Email: $<$ andreas.groll@math.lmu.de>

${ }_{\ddagger}^{\ddagger}$ Ladislaus von Bortkiewicz Chair of Statistics, Humboldt-Universität zu Berlin, Spandauer Straße 1, 10178 Berlin, Germany. Email:< lopezcab@wiwi.hu-berlin.de>

§Mathematical Institute, University of Munich, Theresienstrasse 39, 80333 Munich, Germany. Email: $<$ meyerbra@math.lmu.de> 
information misspecification. Similar to temperature derivatives, this approach can be used for pricing other non-tradable assets.

Key words: factor models, consistency, pricing and hedging, weather derivatives, market price of risk

JEL classification: G19, G29, G22, N23, N53, Q59

\section{Introduction}

In the last years weather derivatives (WD) have emerged to hedge weather variability. This leads to the question how such derivatives are priced and hedged. In contrast to other assets, the pricing of weather derivatives has some challenges since the underlying, contingent on temperature or rain, is not tradeable and the classical Black, Merton and Scholes framework fails since hedging principles cannot be applied. Different streams for pricing weather derivatives are found in the literature: econometric modeling of the underlying dynamics ([12], [10]) followed by risk neutral pricing, equilibrium models [13], indifference pricing models [11], index modeling and burn analysis [23].

In the context of no-arbitrage pricing, there has been some work to calibrate and study the complex structure of the risk premium in temperature derivative prices, or the market price of risk (MPR). The contributions of [13] and [27] study the MPR of weather derivatives as an implicit parameter in a generalization of the Lucas' (1978) equilibrium framework. Equivalent changes of measure with a parametrized time-dependent MPR to get no arbitrage futures/options prices written on different temperature indices were introduced in [10], however the calibration of the MPR was not performed. [22] estimate the MPR from the Taiwan Stock Exchange Capitalization Weighted Stock Index and use it as a proxy for the MPR on temperature option prices.

While most of the papers on temperature derivative pricing impose by assumption a zero or constant MPR ([14], [13], [22], [27] and [1]), a more differentiated analysis conducted in [19], [8], [7], [9] and [20] actually reveals a complex time varying and stochastic behavior of the MPR. However, we believe that at least parts of the irregular behavior of the MPR is not due to irregular risk pricing of the market but due to a misspecification of most models 
that are used to calibrate market prices: forward looking information about the temperature available to the market is not taken into account in the information modeling. The usual assumption that all information available to the market is incorporated in the underlying, i.e. the information filtration is generated by the underlying, might be acceptable for storable assets (classical financial markets). However, for non-storable underlyings (like temperature or electricity) this assumption is fundamentally wrong: lots of meteorological forward looking information available to the market is not reflected in the past evolution of temperature. Hence, an appropriate model for the pricing of temperature derivatives should take into account the information about meteorological forecasts available to the market participants.

There is only few literature dealing with the incorporation of meteorological forecast into weather derivative pricing. [1] suggests, without a model, to incorporate forecasts for shortterm pricing. How to use single and ensemble forecasts to derive probabilistic weather forecasts for weather derivative pricing is described in [23]. [31] incorporates the seasonal forecast in the temperature process, by assuming the unconditional mean temperature as a linear combinations of above-normal (warm), near-normal, and below-normal (cool) mean temperature processes. [6] apply the theory of enlargement of filtration to describe all information available in the market and with it estimate information premiums for given filtrations strictly bigger than the one generated by the underlying. The model from [6] is applied in [28]. There, assuming a MPR equal to zero, it is shown that incorporating weather forecast gives better accurates of market prices. [18] also used an enlargement of the filtration set with weather forecasts by modeling the temperature dynamics process with a larger time series that consists of historical and weather forecast data. Another empirical study of the information premium is described in [5]. The challenge of working with the theory of enlargement of filtrations is the analytic tractability of the forward looking information. In [14] an index modeling approach is used and it is also shown that weather forecasts significantly influence prices. [21] models temperature forecasts by a finite dimensional factor model and guarantees consistency with the martingale dynamics of temperature forecasts. However, no empirical analysis is shown there.

The idea in [21] is two include forward looking information available in the market into temperature modeling by specifying a model for the complete meteorological temperature fore- 
cast curve. More precisely, given a filtered probability space $\left(\Omega, \mathcal{F}, \mathcal{F}_{t \geq 0}, \mathbb{P}\right)$ fulfilling the usual conditions, it is assumed that the meteorological forecast $f(t ; T)$ at time $t$ of the temperature $\tau(T)$ at time $T$ is an unbiased estimator of the temperature in the sense that

$$
f(t ; T)=\mathrm{E}\left[\tau(T) \mid \mathcal{F}_{t}\right]
$$

For fixed forecast time $T$, the forecast process $f(\cdot ; T)$ is thus a martingale under the real world probability measure $\mathbb{P}$ with respect to the flow of available information $\mathcal{F}_{t}$. Next, the evolution of the forecast curves is modeled by a factor model

$$
f(t, T)=H(T-t, Z(t))
$$

where

- $H(x, z): \mathbb{R}_{+} \times \mathbb{R}^{m} \rightarrow \mathbb{R}$ is a given curve family;

- $x=T-t$ is time to forecast time;

- $Z(t)$ is an $\mathbb{R}^{m}$-valued factor process given by an Itô diffusion

$$
d Z(t)=b(Z(t)) d t+\sigma(Z(t)) d W(t), \quad Z(0)=z_{0}
$$

with $W(t)$ a $d$-dimensional $\mathcal{F}_{t}$-Brownian motion.

By setting $T=t$, we then obtain the dynamic model for the temperature $\tau(t)=f(t ; t)$ which now is driven by information contained in the complete forecast curve.

A factor model is called consistent if $H(T-t, Z(t))$ is an $\mathcal{F}_{t^{-}}$-martingale for any fixed $T \geq 0$ in accordance with (1). Given a curve family $H(x, z)$, the consistency requirement imposes certain restrictions on admissible drift coefficients $b(z)$ and volatility coefficients $\sigma(z)$ which, for certain types of families $H(x, z)$, are characterized in [21].

The purpose of this paper is to perform an empirical analysis of temperature and temperature forecast data in the context of a specific two-factor model introduced in [21]. To this end 
we adhere to the following modus operandi.

First, a descriptive statistical analysis of the temperature and temperature forecast data is performed. In particular, a functional principal component analysis (FPCA) is applied which provides a natural choice for the number of factors and characterizes curve co-movements in terms of common factors.

Then we introduce the two-factor model and analyze its properties. Based on the previously performed empirical analysis of curve data we conclude that the consistent two-factor model for pricing temperature derivatives explains the stylized facts of historical and forecast temperature curves (seasonality, seasonal variance, exponential decaying autocorrelation) accurately by decomposing the data curve shapes into two factors describing the mean-reversion in the long horizon and tilting or bending of the curves in the short horizon. The two-factor model is within the framework of the Nelson-Siegel curve model, incorporating time-dependent factors for structural changes (namely the impact of additional forward looking information) and seasonality for periodic patterns. It extends other models proposed in the weather derivative literature by including a mean-reversion to a stochastic mean level.

Next, we specify and implement an iterative two-step algorithm for the estimation of our model. The algorithm switches between a joint maximization of the likelihood with respect to the unknown model parameters and a least-squares estimation step of the second factor of the two-factor model.

Finally, the results from [21] are extended, as we derive explicit prices for temperature derivatives written on Cumulative Average Temperature (CAT), Heating Degree Days (HDD) and Cooling Degree Days (CDD) and quantify risk expectations of market participants by analyzing the dynamics of the market price of risk (MPR) of the associated equivalent martingale measure (EMM). With the information available in the market data (historical temperature, meteorological forecast and futures prices), we calibrate the MPR of temperature derivatives (CAT, CDD, HDD) traded at the Chicago Mercantile Exchange (CME). The factor model shows that the behavior of the implied MPR for futures traded in and out of the measurement period is more stable than other estimates such as e.g. obtained in [19]. This confirms that irregularity of the MPR is not due to irregular risk perception but rather due to information 
misspecification. Similar to temperature derivatives, we conclude that this approach can be used for pricing other non-tradable assets.

Our article is structured as follows. In Section 2 we present the empirical analysis for the forecast data. Section 3 introduces the consistent two factor model and presents an estimation algorithm. The pricing of temperature derivatives with the proposed factor model is developed in Section 4, together with the calibration of the MPR. Section 5 concludes the paper. All computations in this paper were carried out in the statistical program $\mathrm{R}([25])$. The temperature and weather derivative data was obtained from Bloomberg and is available in the Risk Data Center (RDC) of the CRC 649 Economic Risk (http://sfb649.wiwi.huberlin.de/).The meteorological forecast data was obtained from WeatherOnline. To simplify notation, in the following dates are denoted with yyyymmdd format.

Throughout the article we will use the following notations for different forecast curves, depending on the context. As already introduced in $(1), f(t ; t+x)$ denotes the forecast curve implied by our factor model at time $t$ of the temperature $\tau(t+x)$ at time $t+x$. The notation $f_{t ; t+x}$ stands for a time series of observed (discrete) forecast data at time points $t$ of the temperature at time $t+x$, while $f_{t}(x)$ is a smooth (continuous) interpolation of the observed forecast time series at time $t$.

\section{Empirical analysis of temperature forecast curves}

In this section we present an empirical analysis of the temperature and point temperature forecast data of the cities New York and Berlin ${ }^{1}$ for the time period 20081229 - 20131003 and 20081229 - 20101202, respectively. The observed forecast at time $t$ of the average temperature at time $t+x$ is denoted by $f_{t ; t+x}, x=0, \ldots, 14$, i.e. each forecast curve consists of the contemporary temperature $(x=0)$ together with the average forecast (which we obtained from the minimum and maximum) of the next 14 days' temperatures. Adjusting the data for measurement errors

\footnotetext{
${ }^{1}$ Data source: New York Laguardia Airport and Tempelhof Airport Meteorological forecast data from WeatherOnline. We thank Dr. Ulrich Römer and Herrad Werner for providing us the data. Note here that as our forecast and temperature data had to be obtained from different providers, this bears a risk of inconsistencies in the data.
} 
and missing values ${ }^{2}$, we end up with 1740 entire forecast curves for New York and 411 entire forecast curves for Berlin. Figure 1 illustrates the structure of the temperature data together with the forecast curves of three selected days.
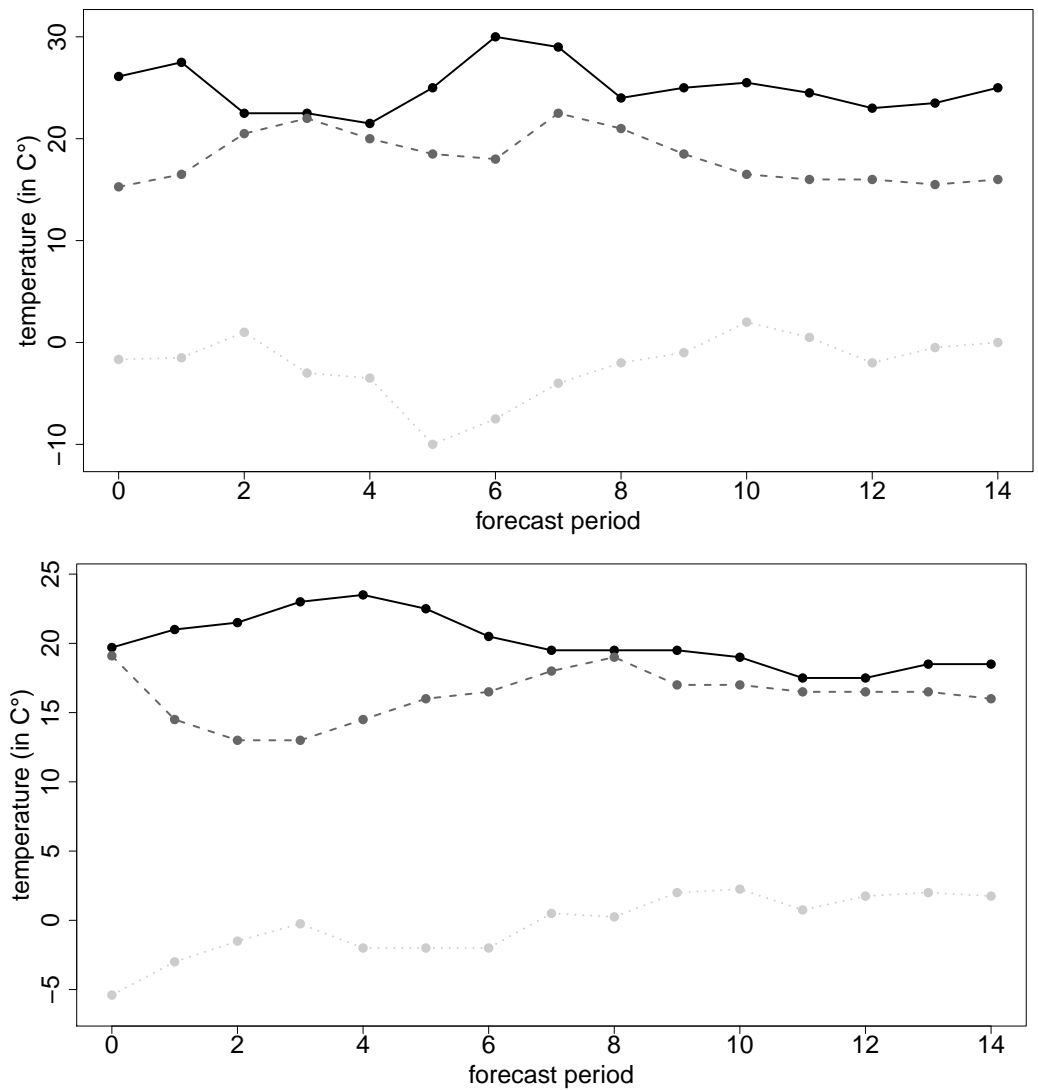

Figure 1: Temperature data $(x=0)$ together with the forecast curves $(x=1, \ldots, 14)$ of three selected days $(t=20090111,20090526,20090804))$ for New York (top) and Berlin (bottom).

\section{$2.1 \quad$ Unbiasedness}

As already stated in the introduction, a central issue in this article is to incorporate forward looking information available in the market by specifying a model for the complete forecast curve. In our approach we assume that the forecast is unbiased in the sense that it represents the expected temperature given all available information (see Equation (1)). We first analyze if this assumption is confirmed in our time series by comparing expected forecasts with expected

\footnotetext{
${ }^{2}$ For New York on 11 days and for Berlin on 6 days the entire forecast curve was missing; here we imputed, if possible, the forecast of a weather station nearby or by averaging over the preceding and subsequent day. For some other days the forecasts of the last or the last two days of the forecast period were missing; here we imputed by the forecast of the preceding day.
} 
temperatures. We use the average differences of true temperatures and forecasts for the different forecast horizons $x$, i.e.

$$
\overline{D_{x}}:=\frac{1}{N_{x}} \sum_{i=1}^{N_{x}} f_{t_{i} ; t_{i}+x}-f_{t_{i}+x ; t_{i}+x}, \quad x=1, \ldots, 14
$$

where $N_{x}$ denotes the number of available pairs of true temperatures and corresponding forecasts for these temperatures. While for Berlin the differences seem to vary randomly around zero, for New York the forecasts seem to slightly overestimate the true temperature, which in fact indicates that there are some inconsistencies in the New York data as already suspected in Footnote 1. Additionally, in order to assess the general predictive quality of the forecasts, we also present the average of the absolute differences for different forecast horizons, i.e.

$$
\overline{\left|D_{x}\right|}:=\frac{1}{N_{x}} \sum_{i=1}^{N_{x}}\left|f_{t_{i} ; t_{i}+x}-f_{t_{i}+x ; t_{i}+x}\right|, \quad x=1, \ldots, 14
$$

As expected, $\overline{\left|D_{x}\right|}$ is generally increasing for larger forecast horizons, compare Table 1 , but is still in a moderate range for both cities. Though for Berlin the forecasts seem less biased, in summary we can say that for both cities the forecasts serve as proper predictions of the true temperatures.

\begin{tabular}{llrrrrrrrrrrrrrr}
\hline & $x$ & 1 & 2 & 3 & 4 & 5 & 6 & 7 & 8 & 9 & 10 & 11 & 12 & 13 & 14 \\
\hline \multirow{2}{*}{$\overline{D_{x}}$} & New York & .08 & .21 & .29 & .33 & .35 & .44 & .51 & .75 & .99 & 1.14 & 1.25 & 1.34 & 1.42 & 1.45 \\
& Berlin & -.04 & -.06 & .05 & -.02 & .06 & .17 & .15 & -.04 & -.07 & .07 & .22 & .35 & .51 & .60 \\
\hline \multirow{D_{x}|}{*}{} & New York & .95 & 1.15 & 1.31 & 1.52 & 1.70 & 1.95 & 2.16 & 2.36 & 2.63 & 2.79 & 2.90 & 3.05 & 3.13 & 3.20 \\
& Berlin & 1.55 & 1.60 & 1.68 & 1.61 & 1.83 & 1.86 & 2.12 & 2.25 & 2.21 & 2.42 & 2.63 & 2.73 & 2.96 & 3.09 \\
\hline
\end{tabular}

Table 1: Average of standard and absolute differences of true temperatures and forecasts for the different forecast horizons $x$ and different locations.

\subsection{De-seasonalization}

Based on the true average temperatures ${ }^{3}$ of New York - Laguardia airport for the time period 1997-2012 and of Berlin - Tempelhof airport for the time period 1948-2011, a linear trend (for possible temperature increases due to global warming) together with a periodic seasonal trend component have been estimated using non-linear least squares estimation (NLSE), which

\footnotetext{
${ }^{3}$ Minimum, maximum and average temperatures are provided by CME website.
} 
is implemented e.g. in the R-function nls (see [3]) and is based on the following truncated Fourier series

$$
\Lambda(t)=a_{1}+a_{2} t+a_{3} \cos \left(\frac{2 \pi\left(t-a_{4}\right)}{365}\right)
$$

for parameters $a_{k}, k=1, \ldots, 4$ and with time $t$ (daily scale). Note that one could refine this seasonality function by adding more and more periodic terms, but this will increase the number of parameters. Alternatively, non-parametric techniques could be used as described e.g. in [18].

Next, the temperature time series and the 14 forecast time series for the different forecast periods of New York and Berlin are de-seasonalized by subtracting the trend function $\Lambda(t)$ from Equation (2). Exemplarily, the de-seasonalized time series for the true temperature and a forecast period of 6 days are illustrated in Figure 2 for both locations. For the set of forecast curves from Figure 1, the corresponding de-seasonalized forecast curves are shown in Figure 3.
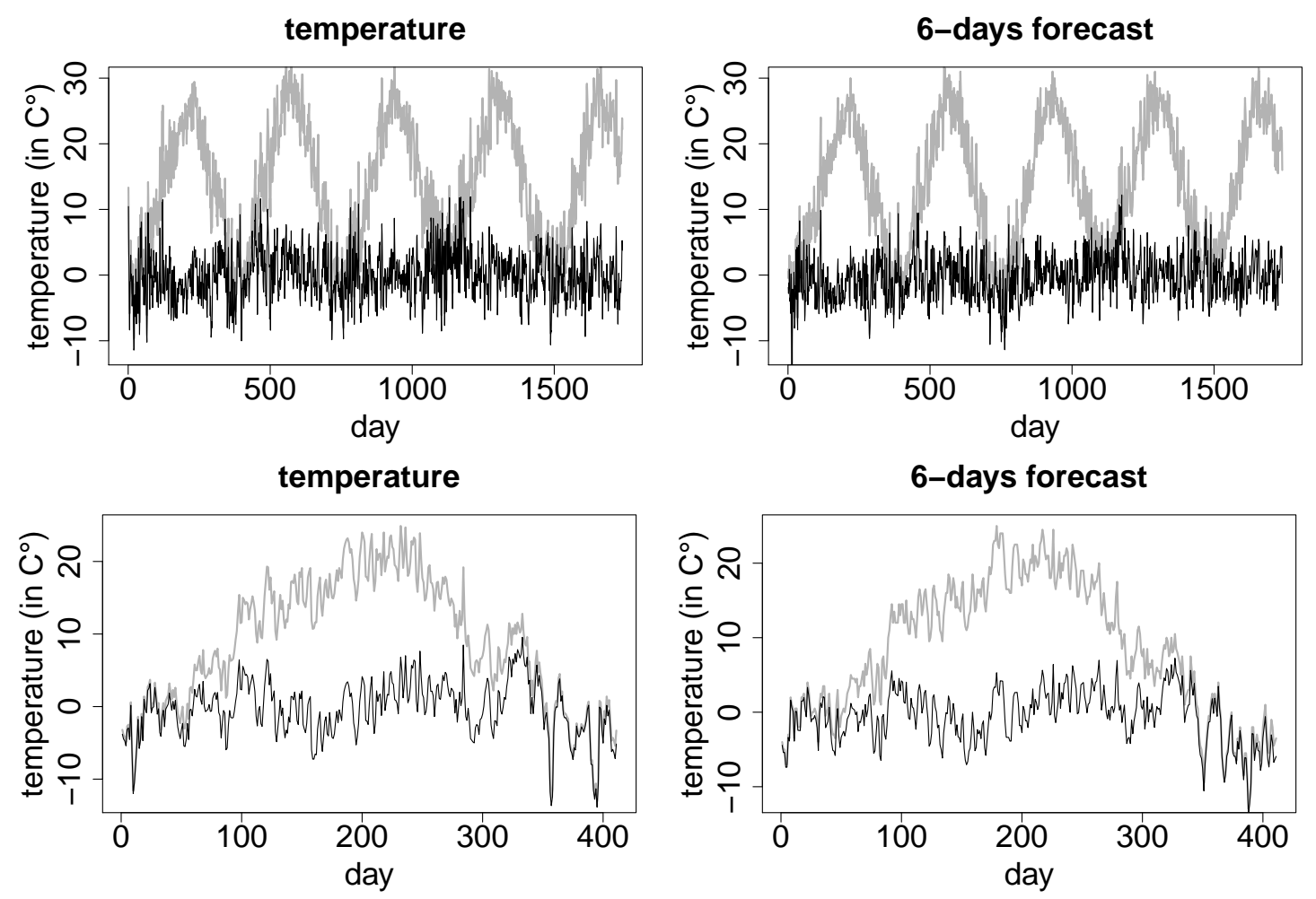

Figure 2: Origninal (gray) and de-seasonalized time series (black) for New York (top) and Berlin (bottom), exemplarily for the true temperature and the "6 days" forecast period. 

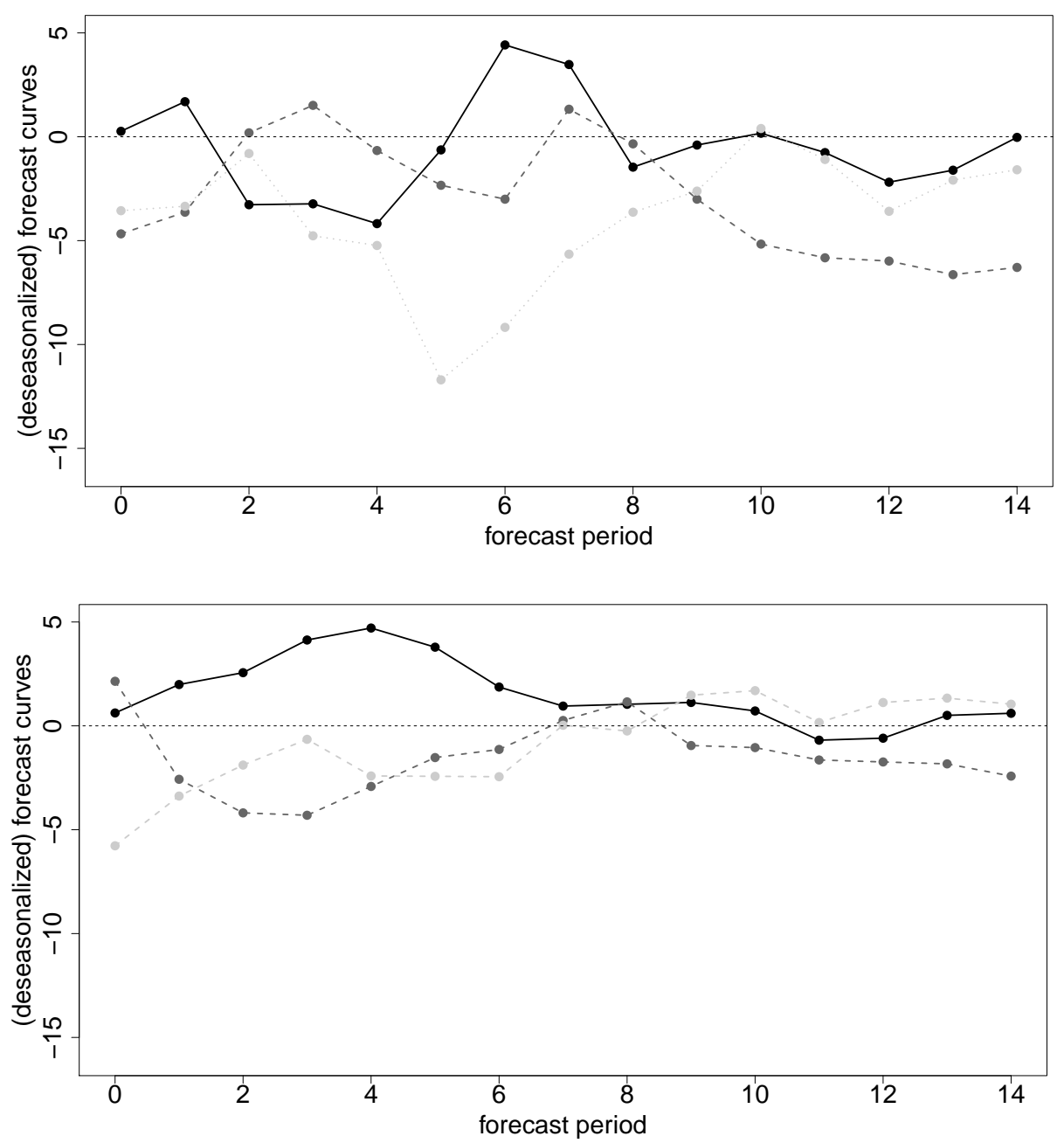

Figure 3: Three de-seasonalized forecast curves for New York (top) and Berlin (bottom).

\subsection{Distributional properties of the forecast data}

Next, we graphically analyze the assumption of normality for the de-seasonalized time series. For both New York and Berlin we found no distinct violations in the corresponding q-q-plots (see Figure 4). In the next step, we apply a conventional statistical test on normality, the Kolmogorov-Smirnov test. It rejects the assumption of normality never for both cities at a significance level of $\alpha=0.01$. Hence, the assumption of normality seems justifiable, which is in accordance with the theoretical properties of the two-factor model presented in Section 3.1.

\subsection{Stationarity and autocorrelation}

In the next step we check for intertemporal autocorrelation and stationarity of the temperature and forecast time series. The partial autocorrelation plots indicate that there is significant 

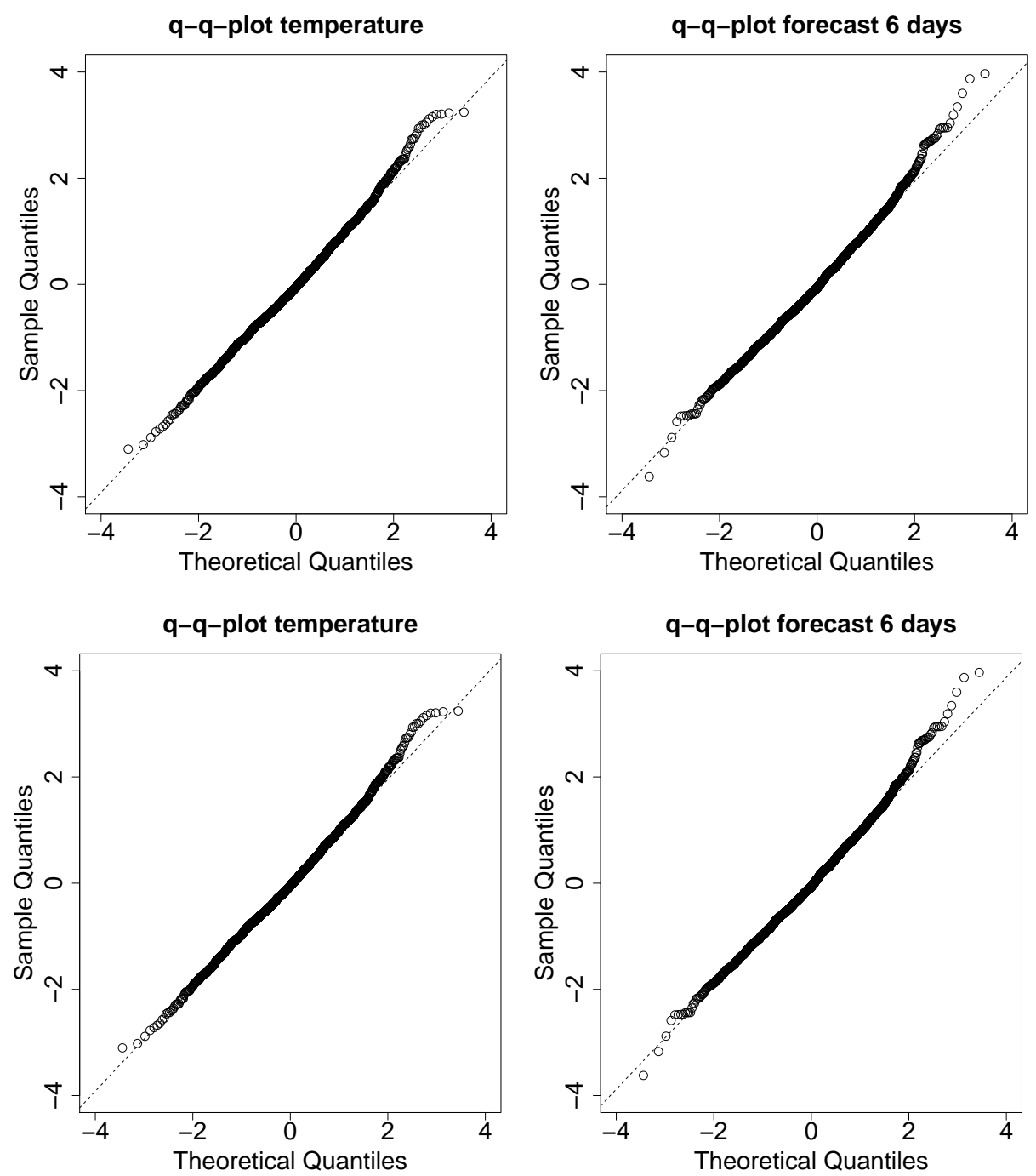

Figure 4: Selected q-q-plots of the de-seasonalized time series (temperature and six day forecast) for New York (top) and Berlin (bottom).

autocorrelation up to about three lags (except for some single artifacts), which is in accordance with the literature e.g. in [10] and [19]. Figure 5 exemplarily shows the partial autocorrelations for the temperature and a forecast period of six days for both locations. Moreover, for New York the corresponding augmented Dickey-Fuller test, implemented in the R-function adf.test, confirms that all 15 time series (true temperature and 14 forecasts) are stationary and the null hypotheses corresponding to the presence of a unit root can be rejected for all tests at a level of significance of $\alpha=0.01$. For Berlin we get similar results and the presence of a unit root is rejected at $\alpha=0.01$ for the true temperature as well as for the forecast horizons $x \in\{1, \ldots, 9\}$. For the forecast horizons $x \in\{10, \ldots, 14\}$ the presence of a unit root is only rejected at a level of significance of $\alpha=0.05$. 

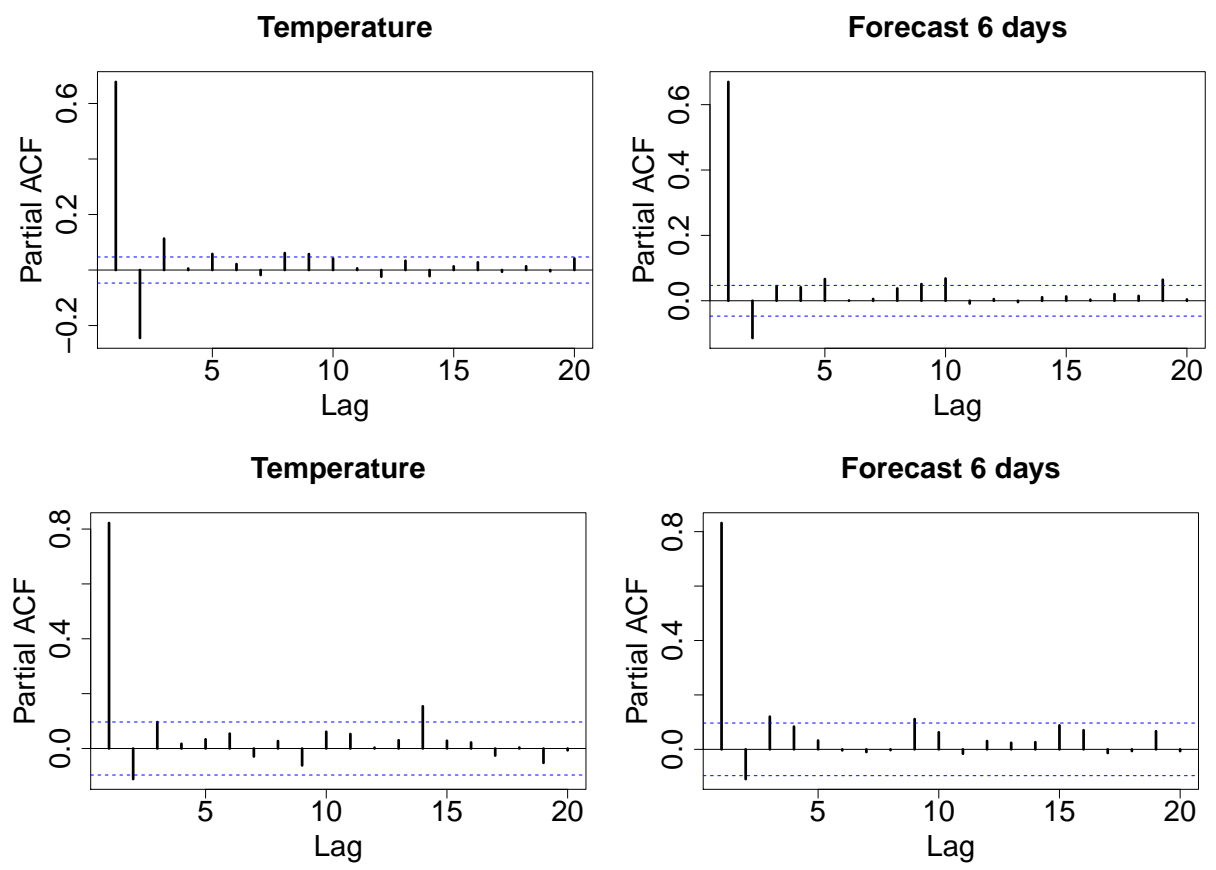

Figure 5: Partial autocorrelations of the temperature and the forecast time series, exemplarily for a forecast period of six days, for New York (top) and Berlin (bottom).

\subsection{Functional principal component analysis}

In this section, we further explore the features characterizing typical (de-seasonalized) forecast curves, in particular we analyze by how many factors the curves are driven. A classical approach in this direction is principal component analysis (PCA), providing an informative way of looking at the covariance structure.

However, in the two-factor model presented in Section 3.1 the forecast curve at a certain time $t$ is considered as a smooth continuous function of the forecast horizon $x$. This suggests to regard our data in a functional context. Furthermore, several authors such as [29] and [30] state that the computation of PCA runs into serious difficulties in analyzing functional data because of the so-called "curse of dimensionality" (see also [4]). Following [29], these difficulties can be overcome by functional principal components analysis (FPCA, i.e. PCA extended to functional data; see [26, Chapter 8.]), which provides a more informative way of examining the sample covariance structure than conventional PCA and which also complements a direct examination of the variance-covariance structure and characterizes curve comovements in terms of common factors. Besides, if necessary, FPCA allows to incorporate regularization.

Consequently, we transform all 1740 forecast curves of New York and all 411 forecast curves 
of Berlin into smooth functional data objects using a penalized basis function approach described in [26], which is implemented in the R-package fda. Note here that also the functional mean of a set of $N$ different functions can be derived following [26], who provide a mean() method for functional data objects using

$$
\bar{g}(t)=N^{-1} \sum_{i=1}^{N} g_{i}(t)
$$

in their R-package fda. Figure 6 shows the de-seasonalized forecast data together with the corresponding smoothed forecast curves (gray) and their functional mean (black), which is close to the zero-function, exemplarily for New York; for Berlin we obtain a very similar graph.

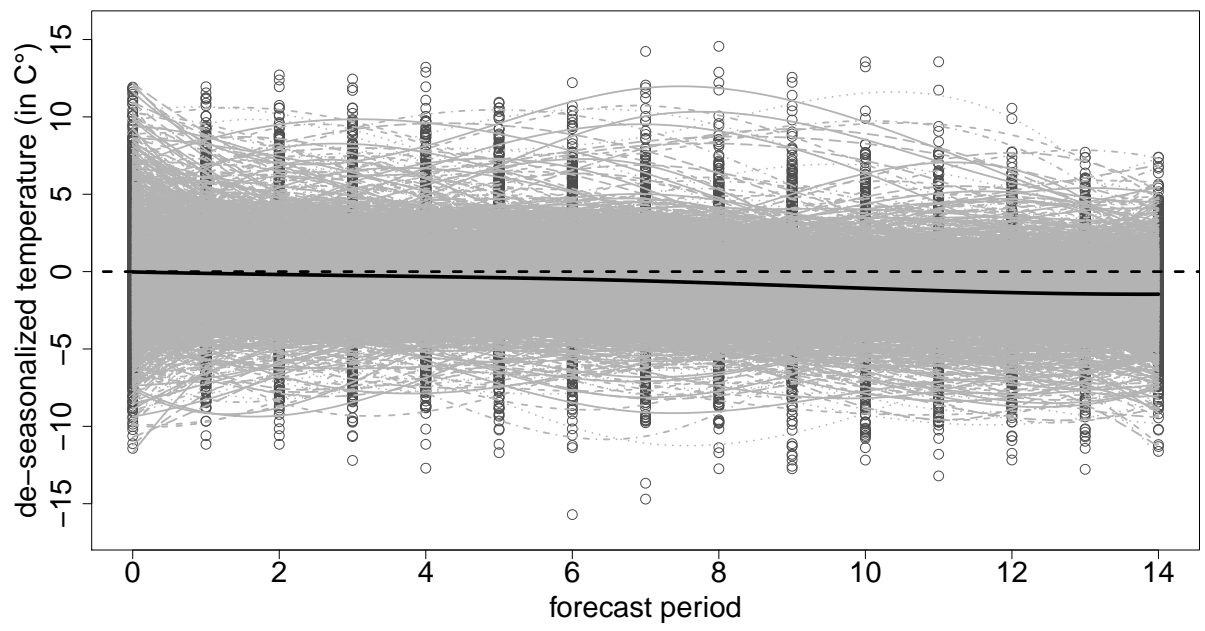

Figure 6: De-seasonalized forecast data together with the smoothed forecast curves (gray) and their functional mean (black) for New York.

Now we perform a FPCA based on our smooth forecast curves, which we denote by $f_{t}(x), t=$ $1, \ldots, N, x \in[0,14]$. To start with, we need an appropriate definition of the inner product for functions. In conventional PCA for multivariate data the focus is on choosing those weights $\beta$ (loadings), which maximize the average variation in

$$
h_{i}=\beta^{\top} \mathbf{f}_{i}=\sum_{j} \beta_{j} f_{i j}, \quad i=1, \ldots, N
$$

for given data vectors $\mathbf{f}_{i}$. If instead $\beta(x)$ is a weight function and $f_{i}(x)$ a functional data object, now summations over $j$ are replaced by integrations over $x$ and the inner product is then 
defined by $\int \beta(x) f_{i}(x) d x$, where the integral is defined over the range of $x$ (as we consider 14 days forecasts together with the contemporary temperature, we have $x \in[0,14])$. Consequently, within functional PCA, for a given weight function $\beta(x)$ the corresponding principal component score is given by

$$
h_{i}=\int \beta(x) f_{i}(x) d x
$$

In the first FPCA step, a weight function $\beta_{1}(x)$ is chosen to maximize the sum

$$
\frac{1}{N} \sum_{i} h_{i 1}^{2}=\frac{1}{N} \sum_{i}\left(\int \beta_{1}(x) f_{i}(x) d x\right)^{2}
$$

subject to the constraint $\left\|\beta_{1}\right\|^{2}:=\int \beta_{1}(x)^{2} d x=1$, which is the continuous analog of the unit sum of squares constraint used in the conventional multivariate PCA. Similar to multivariate $\mathrm{PCA}$, this procedure is carried out in subsequent steps for further weight functions $\beta_{m}(x), m=$ $2, \ldots, p$, each satisfying the orthogonality constraint $\int \beta_{k}(x) \beta_{m}(x) d x=0, k<m$, up to a maximum of $p$ weight functions, which in our case corresponds to the number of basis functions determined in the penalized basis function approach. Hence, each weight function defines the most important mode of variation in the curves subject to each mode being orthogonal to all modes defined on previous steps. For the computational details concerning the FPCA we refer to $[26$, Chapter 8.4].

The results of the FPCA on our smooth forecast curves are illustrated in Figure 7. It can be seen that the first two functional principle components already account for $78.1 \%$ of the total variation for the New York data and even for $88.5 \%$ for the Berlin data. For both locations, the first weight function $\beta_{1}(x)$ represents a simple tilting feature, whereas the second weight function $\beta_{2}(x)$ allows for bending, i.e. the forecast curves can also take course of a hump before returning to the mean. These results provide the statistical justification that a two factor model for the specification of 14-days forecast curves, as proposed in the next section, is a reasonable choice. 
PC 1 (52.8\%)

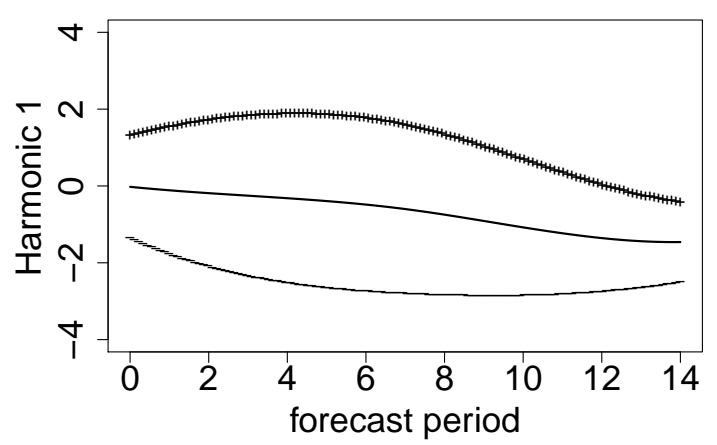

PC 1 (69.7\%)

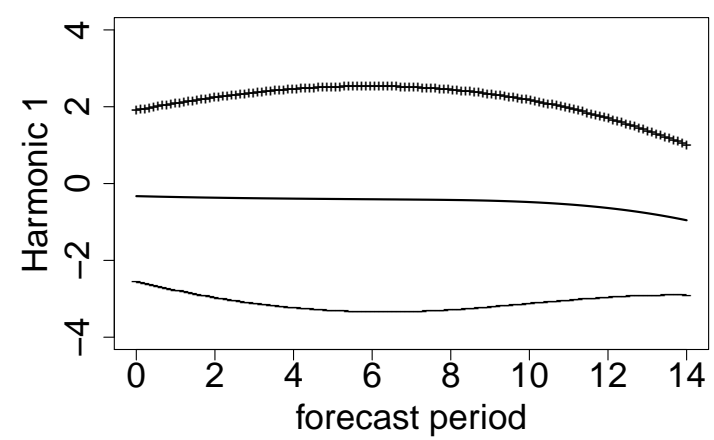

PC 2 (25.3\%)

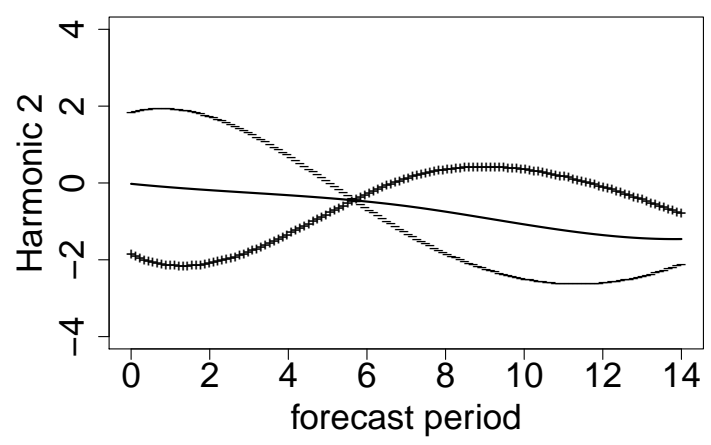

PC 2 (18.8\%)

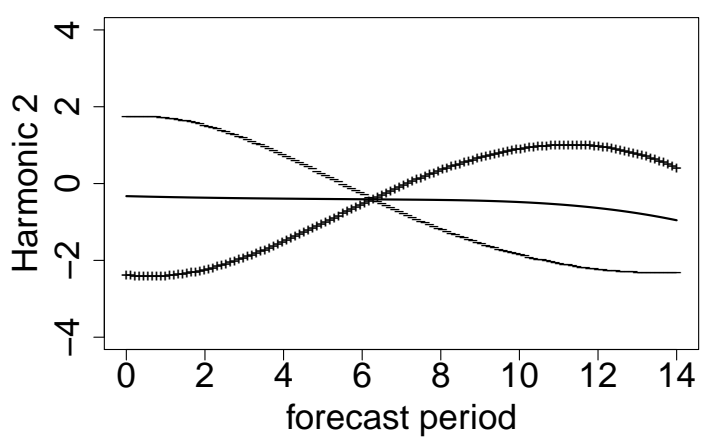

Figure 7: The mean de-seasonalized forecast curve and the effect of adding $(+)$ and subtracting (-) a suitable multiple of the first two weight functions $\beta_{i}(x)$ for New York (top) and Berlin (bottom); proportion of explained variation in brackets.

\subsection{Summary of the stylized features}

Summing up, our empirical analysis exhibits the following stylized features of the forecast curves, both for the New York and Berlin data.

- In terms of average standard and absolute differences the forecasts seem to have quite good predictive power with respect to the true temperatures, at least for small forecast horizons.

- In general, the assumption of normality seems justifiable for the de-seasonalized forecasts (including the contemporary temperature for $x=0$ ) with regard to q-q-plots and a standard statistical test on normality.

- We found significant autocorrelation up to the third lag in the time series of all deseasonalized forecasts and, additionally, unit root tests confirmed that the time series are also stationary for all forecast horizons $x \in\{0, \ldots, 14\}$. 
- The results of a functional principal component analysis suggest a two factor model for the specification of 14-days forecast curves, the first weight function representing tilting and the second weight function allowing for bending.

In the next section a suitable two factor temperature forecast curve model is proposed, together with a procedure for the estimation of the corresponding model parameters.

\section{Estimating a consistent two-factor model}

Based on the empirical analysis of the New York and Berlin forecast curve data, we will now propose a suitable factor model and explain an estimation procedure for this model.

\subsection{The model}

In accordance with our findings in the functional principal component analysis in Section 2.5, we propose a two-factor temperature forecast curve model that was introduced in [21]. In this model, the forecast at time $t$ of the temperature at time $t+x(x=T-t$ is time to forecast) is given by

$$
\begin{aligned}
f(t ; t+x) & =\Lambda(t+x)+H(x, Z(t)) \\
& :=\Lambda(t+x)+Z_{1}(t) e^{-\lambda x}+Z_{2}(t) \frac{1}{\lambda-\rho}\left(e^{-\rho x}-e^{-\lambda x}\right)
\end{aligned}
$$

or re-parametrized in terms of forecast time $T=x+t$ we get

$$
f(t ; T)=\Lambda(T)+Z_{1}(t) e^{-\lambda(T-t)}+Z_{2}(t) \frac{1}{\lambda-\rho}\left(e^{-\rho(T-t)}-e^{-\lambda(T-t)}\right)
$$

where $\Lambda(t)$ is a deterministic seasonality function (average temperature at time $t$ ) and

$$
H(x, z)=z_{1} e^{-\lambda x}+z_{2} \frac{1}{\lambda-\rho}\left(e^{-\rho x}-e^{-\lambda x}\right)
$$


$H: \mathbb{R}_{+} \times \mathbb{R}^{2} \rightarrow \mathbb{R}$ determines the essential features of our forecast curve family. The model for the two-dimensional factor process

$$
Z(t):=\left(Z_{1}(t), Z_{2}(t)\right)
$$

is a $\mathbb{R}^{2}$-valued factor process given in (6)-(7) below. For the de-seasonalized forecast curves $\tilde{f}(t ; t+x)=f(t ; t+x)-\Lambda(t+x)$ model (3) yields

$$
\tilde{f}(t ; t+x)=Z_{1}(t) e^{-\lambda x}+Z_{2}(t) \frac{1}{\lambda-\rho}\left(e^{-\rho x}-e^{-\lambda x}\right) .
$$

Choosing $x=0$ in (3), we see that the role of the first factor $Z_{1}(t)$ is to model the deseasonalized contemporary temperature: $Z_{1}(t)=\tilde{f}(t ; t)=\tau(t)-\Lambda(t)$. The knowledge of the past temperature at time $t$ contributes to the forecast curve by an exponential mean-reversion from current temperature levels towards the seasonal average temperature induced by the component $Z_{1}(t) e^{-\lambda x}$. Following the popular approach to model the temperature by an OrnsteinUhlenbeck process and to let the information filtration be generated by the temperature (see for example [10]), the implied forecast curves would be of this exponentially decaying type, which, however, does not give a good fit to the family of empirically observed forecast curves (see Figure 11 in Section 3.2). That is why we introduce a second factor $Z_{2}(t)$ which is responsible for modeling the additionally available forward looking information on the temperature. At time $t$, it contributes with the component $Z_{2}(t) \frac{1}{\lambda-\rho}\left(e^{-\rho x}-e^{-\lambda x}\right)$ to the forecast curve and might produce humps or dips in the curve (see Figure 8).

The two curve components in our model are motivated by the fact that they exhibit the qualitative behavior of the first two weight functions obtained from the FPCA in Figure 7. See Figure 8 for some typical (de-seasonalized) curves produced by our model. Note that (4) is a generalization of the popular Nelson-Siegel curve family in interest rate modeling (see e.g. [24], [15]), as for $\lambda \rightarrow \rho$ our factor model $H(x, Z(t))$ converges point-wisely to the NelsonSiegel model without parallel shift parameter. The reason we consider this generalization is that then the consistent factors $Z_{1}(t)$ and $Z_{2}(t)$ in (6)-(7) below are allowed to have different mean-reversion parameters $\lambda \neq \rho$ while in the Nelson-Siegel model these must be identical. 


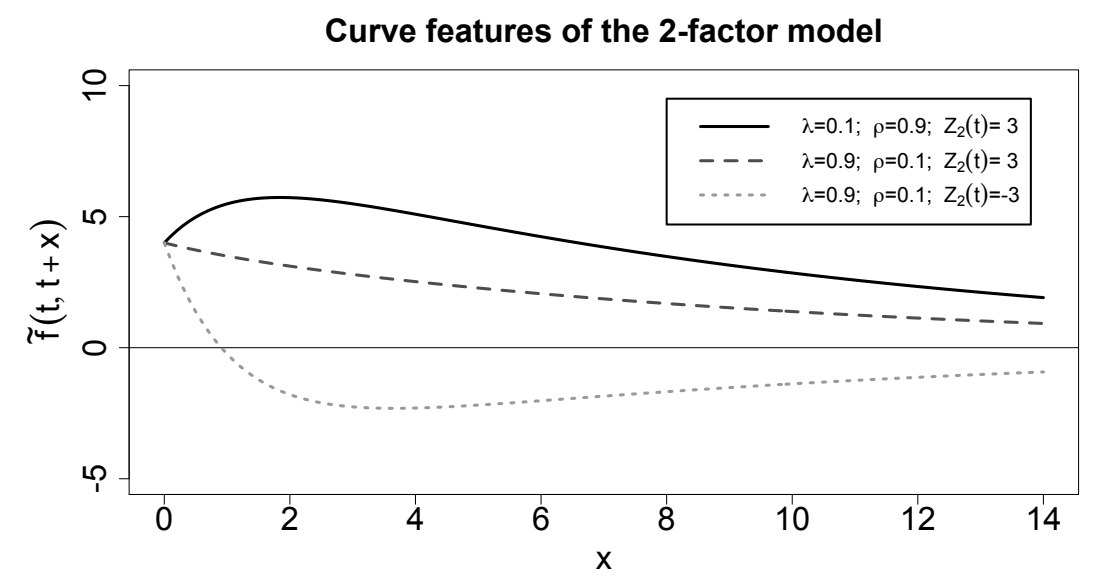

Figure 8: Typical curve features of the de-seasonalized forecast curves $\tilde{f}(t ; t+x)$ from (5) for different choices of $\lambda, \rho$ and $Z_{2}(t)$ for New York.

To complete our forecast curve model it remains to specify the dynamics of the factor process $Z(t):=\left(Z_{1}(t), Z_{2}(t)\right)$ under the restriction that $Z(t)$ is consistent with the forecast curve family $H(x, z)$, see Introduction. We propose the following two-dimensional Itô-diffusion which is shown to be consistent in [21]:

$$
\begin{aligned}
& d Z_{1}(t)=\left(-\lambda Z_{1}(t)+Z_{2}(t)\right) d t+\sigma_{1}(t) d W_{1}(t) \\
& d Z_{2}(t)=-\rho Z_{2}(t) d t+\sigma_{2} d W_{2}(t)
\end{aligned}
$$

where $\lambda, \rho, \sigma_{2}>0, \sigma_{1}(t)$ is a deterministic and bounded volatility function, and $W_{1}(t)$ and $W_{2}(t)$ are independent $\mathbb{P}$-Brownian motions.

We assume that the underlying information filtration $\left\{\mathcal{F}_{t}\right\}_{t \geq 0}$ is the one generated by $W_{1}$ and $W_{2}$. Actually, in [21] it is shown that any consistent two-dimensional Itô-diffusion necessarily has the drift given in (6)-(7), while the volatility can be chosen freely subject to some integrability restrictions. Note that adding the deterministic quantity $\Lambda(T)$ to $H(T-t, Z(t))$ does not change the martingale property such that the model remains consistent.

Our volatility choice $\sigma_{1}(t)$ is motivated by the analysis in [10], where the authors conclude that a deterministic but seasonally varying volatility is appropriate for the de-seasonalized temperature $Z_{1}$. If $Z_{2}(t)=0$, the temperature model in (6) would be the same as the one proposed in [10]. However, compared to the model in [10], the dynamics of the de-seasonalized temperature $Z_{1}$ implied by our forecast curve model are those of an extended Ornstein-Uhlenbeck 
process with a stochastic mean-reversion level which is governed by the factor $Z_{2}$ and integrates additional forward looking information contained in meteorological temperature forecasts. The factor $Z_{2}$ follows a regular Ornstein-Uhlenbeck process, where we chose a constant volatility since forecast curve time series are not long enough to infer further volatility structures of $Z_{2}$.

Next, we analyze the distributional properties of our factor process. In particular, since it is well known that the Itô-diffusion $Z$ is a two-dimensional Markov process, we are interested in the conditional distribution of $Z(s)$ given $Z(t), 0 \leq t \leq s$, in order to build an appropriate maximum-likelihood-estimation scheme to estimate the model in Section 3.2.

The analytic solution of the Ornstein-Uhlenbeck process $Z_{2}$ in $(7)$ for $0 \leq t \leq s$ is given by

$$
Z_{2}(s)=Z_{2}(t) e^{-\rho(s-t)}+\int_{t}^{s} \sigma_{2} e^{-\rho(s-u)} d W_{2}(u)
$$

Hence, with the quadratic variation of Itô-processes, for $s \geq t \geq 0$ the conditional distribution of $Z_{2}(s)$ given $Z(t)$ is

$$
Z_{2}(s) \mid Z(t) \sim \mathrm{N}\left(Z_{2}(t) e^{-\rho(s-t)}, \frac{\sigma_{2}^{2}}{2 \rho}\left(1-e^{-2 \rho(s-t)}\right)\right)
$$

Similarly, from (6) we obtain a closed form solution for the first factor $Z_{1}$ at time $s \geq t \geq 0$ :

$$
Z_{1}(s)=Z_{1}(t) e^{-\lambda(s-t)}+\int_{t}^{s} Z_{2}(u) e^{-\lambda(s-u)} d u+\int_{t}^{s} \sigma_{1}(u) e^{-\lambda(s-u)} d W_{1}(u)
$$

Inserting (8) and employing stochastic Fubini, we obtain for the second summand

$$
\begin{aligned}
& \int_{t}^{s} Z_{2}(u) e^{-\lambda(s-u)} d u \\
& =\int_{t}^{s}\left\{Z_{2}(t) e^{-\rho(u-t)}+\int_{t}^{u} \sigma_{2} e^{-\rho(u-z)} d W_{2}(z)\right\} e^{-\lambda(s-u)} d u \\
& =Z_{2}(t) \int_{t}^{s} e^{-\rho(u-t)-\lambda(s-u)} d u+\int_{t}^{s} \int_{z}^{s} \sigma_{2} e^{-\rho(u-z)-\lambda(s-u)} d u d W_{2}(z) \\
& =Z_{2}(t) \frac{e^{-\rho(s-t)}-e^{-\lambda(s-t)}}{\lambda-\rho}+\int_{t}^{s} \sigma_{2} \frac{e^{-\rho(s-z)}-e^{-\lambda(s-z)}}{\lambda-\rho} d W_{2}(z) .
\end{aligned}
$$


Hence, for $s \geq t \geq 0$ we compute the conditional distribution of $Z_{1}(s)$ given $Z(t)$ to be

$$
Z_{1}(s) \mid Z(t) \sim \mathrm{N}\left(\mu_{t, s} ; \psi_{t, s}^{2}\right)
$$

where

$$
\mu_{t, s}=Z_{1}(t) e^{-\lambda(s-t)}+Z_{2}(t) \frac{e^{-\rho(s-t)}-e^{-\lambda(s-t)}}{\lambda-\rho}
$$

and

$$
\begin{aligned}
\psi_{t, s}^{2} & =\int_{t}^{s} \sigma_{1}^{2}(u) e^{-2 \lambda(s-u)} d u \\
& +\frac{\sigma_{2}^{2}}{(\lambda-\rho)^{2}}\left\{\frac{1-e^{-2 \rho(s-t)}}{2 \rho}+\frac{1-e^{-2 \lambda(s-t)}}{2 \lambda}-\frac{2\left(1-e^{-(\rho+\lambda)(s-t)}\right)}{\rho+\lambda}\right\}
\end{aligned}
$$

From (8) and (9) we see that for $s \geq t \geq 0$ the conditional distribution of $Z(s) \mid Z(t)$ is a two-dimensional Gaussian distribution since it is the distribution of a linear transformation of a two-dimensional vector of independent Gaussian random variables. To specify this twodimensional Gaussian distribution it remains to determine the covariance:

$$
\begin{aligned}
\operatorname{Cov}\left(Z_{1}(s), Z_{2}(s) \mid Z(t)\right) \\
=\mathrm{E}\left[\left(Z_{1}(s)-\mathrm{E}\left[Z_{1}(s) \mid Z(t)\right]\right)\left(Z_{2}(s)-\mathrm{E}\left[Z_{2}(s) \mid Z(t)\right]\right) \mid Z(t)\right] \\
=\mathrm{E}\left[( \int _ { t } ^ { s } \sigma _ { 2 } e ^ { - \rho ( s - u ) } d W _ { 2 } ( u ) ) \left(\int_{t}^{s} \sigma_{2} \frac{e^{-\rho(s-u)}-e^{-\lambda(s-u)}}{\lambda-\rho} d W_{2}(u)\right.\right. \\
\left.\left.\quad+\int_{t}^{s} \sigma_{1}(u) e^{-\lambda(s-u)} d W_{1}(u)\right) \mid Z(t)\right] \\
=\mathrm{E}\left[\left(\int_{t}^{s} \sigma_{2} e^{-\rho(s-u)} d W_{2}(u)\right)\left(\int_{t}^{s} \sigma_{2} \frac{e^{-\rho(s-u)}-e^{-\lambda(s-u)}}{\lambda-\rho} d W_{2}(u)\right) \mid Z(t)\right] \\
=\int_{t}^{s} \frac{\sigma_{2}^{2}}{\lambda-\rho} e^{-\rho(s-u)}\left(e^{-\rho(s-u)}-e^{-\lambda(s-u)}\right) d u \\
=\frac{\sigma_{2}^{2}}{\lambda-\rho}\left(\frac{1-e^{-2 \rho(s-t)}}{2 \rho}-\frac{1-e^{-(\rho+\lambda)(s-t)}}{\rho+\lambda}\right):=c_{s-t}
\end{aligned}
$$


Finally, we end up with

$$
\left(\begin{array}{c}
Z_{1}(s) \\
Z_{2}(s)
\end{array}\right) \mid Z(t) \sim \mathrm{N}_{2}\left(\left(\begin{array}{c}
\mu_{t, s} \\
Z_{2}(t) e^{-\rho(s-t)}
\end{array}\right),\left(\begin{array}{cc}
\psi_{t, s}^{2} & c_{s-t} \\
c_{s-t} & \frac{\sigma_{2}^{2}}{2 \rho}\left(1-e^{-2 \rho(s-t)}\right)
\end{array}\right)\right)
$$

for $s \geq t \geq 0$.

Summing up, the proposed forecast curve model exhibits the following features reflecting the outcomes of the empirical analysis in Section 2:

- Forecast curves are built from two components that feature the qualitative behavior of the first two weight functions obtained from the FPCA. This type of qualitative forecast curve formation catches most of the total variation.

- As the forecast horizon $T-t$ gets large, the volatility of forecast temperature diminishes and

$$
f(t ; T) \rightarrow \Lambda(T) \text { for }(T-t) \rightarrow \infty .
$$

This behavior is realistic since forward looking information decreases the larger the forecast horizon becomes, and finally average temperature is the best prediction.

- Before reverting to the seasonal average temperature in the long end, there are basically two different types of qualitative behavior of forecast curves in the shorter end corresponding to the two components:

1. Direct exponential reversion in temperature forecasts from the current temperature level $f(t ; t)$ to the seasonal function $\Lambda(T)$.

2. An increase or decrease (hump or dip) from $f(t ; t)$ in forecast temperature prior to exponential reversion to the seasonal function $\Lambda(T)$.

- For fixed forecast time $T>0$, the forecast $f(t ; T)$ is a martingale (i.e. the model is consistent), and hence, an unbiased estimate for the true temperature. This is also in accordance with our findings in Section 2.1.

- From (12) and (5) we see that de-seasonalized forecasts $\tilde{f}(t ; t+x)$ (including the contemporary de-seasonalized temperature $\tilde{f}(t ; t))$ are Gaussian, which is again generally 
consistent with the results from Section 2.3.

- It is well known that (for the right initial value and constant volatility $\sigma_{1}$ ) $\mathrm{Z}$ is a stationary process. It follows from (5) that for a fixed time to forecast $x$, the de-seasonalized forecasts $\tilde{f}(t ; t+x)$ are stationary processes.

- The imposed evolution of the forecast curves implies that the de-seasonalized temperature $Z_{1}$ in (6) follows an extended Ornstein-Uhlenbeck process that is mean-reverting to the stochastic level $\frac{1}{\lambda} Z_{2}(t)$. In this way, additional forward looking information represented by $Z_{2}$ impacts the modeling of future temperature.

\subsection{Model estimation}

Given a time series $\tilde{f}_{t ; t+x}, t=1, \ldots, N$, of observed (de-seasonalized) forecast curves, we present a two-step algorithm to estimate the model on these data. More precisely, we iteratively repeat the following two steps until convergence of the estimated parameters:

- In the first step we construct a two-dimensional time series $\left(\hat{Z}_{1}(t), \hat{Z}_{2}(t)\right), t=1, \ldots, n$, corresponding to realizations of our factor process $Z=\left(Z_{1}, Z_{2}\right)$. We recall that $\hat{Z}_{1}(t)$ is observable since $Z_{1}(t)$ represents the contemporary (de-seasonalized) temperature, and thus the interest in this step is to filter the time series $\hat{Z}_{2}(t), t=1, \ldots, n$, which we do with the help of a least-squares- (LS-)method (except for getting initial values; this is done via a differential evolution algorithm).

- In the second step, given the time series $\left(\hat{Z}_{1}(t), \hat{Z}_{2}(t)\right), t=1, \ldots, N$, we estimate the parameters in our model, which are $\rho, \lambda, \sigma_{2}$ and the deterministic process $\sigma_{1}(t)$, by the maximum-likelihood- (ML-)method.

We now describe in more detail the procedure, exemplarily for the New York data. The results for the Berlin data are shortly summarized at the end of this section. In the following we define for $s=t+1$ in (11)

$$
\psi^{2}(t):=\psi_{t, t+1}^{2} \quad, t \geq 0
$$




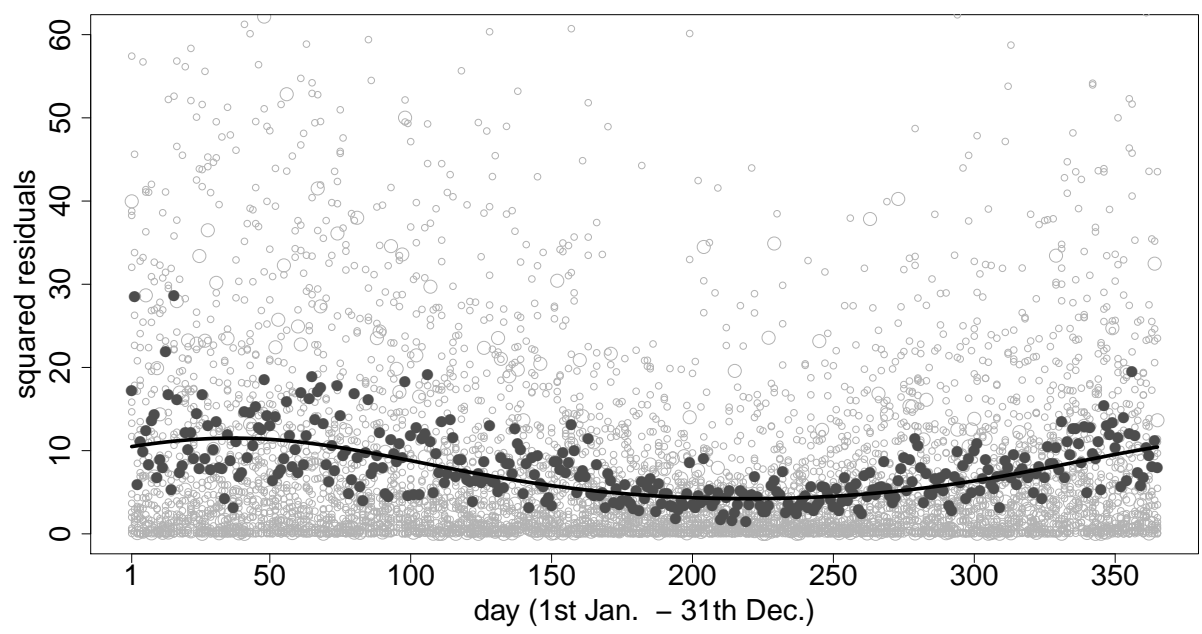

Figure 9: New York squared $A R(1)$-residuals (light gray) for the time period 1997-2012, average squared residuals over all 16 years (dark gray) together with a non-linear least squares estimate of $\psi^{2}(t)$ based on a truncated Fourier series approximation (black solid line).

First, we need reasonable starting values for the factor $Z_{2}(t)$ and for the parameters $\rho$ and $\lambda$. These can be obtained by use of a differential evolution- (DE-)algorithm, following [16], who showed that the DE-algorithm is capable of reliably solving the Nelson-Siegel-Svennson model. An implementation of the DE-algorithm is available in the R-package NMOF, see [17]. Note that together with the package, a very helpful vignette called "Fitting the Nelson-Siegel-Svensson model" is available.

A parametric smooth starting estimate for the process $\psi(t)$ can be obtained by the following strategy. Based on New York's average temperatures for the time period 1997-2012 we fit an $A R(1)$-model (autoregressive model of order 1), compute the corresponding squared residuals and average them over the 16 years. Using again the NLSE technique from Section 2.2, the (average) squared residuals are approximated by a truncated Fourier series ${ }^{4}$, compare Figure 9 (black solid line), yielding optimal Fourier coefficients $\gamma$ as starting values. Note that ACFplots of the squared residuals do not show signs of stochastic volatility: the squared residuals do not have an exponentially decaying ACF, compare Figure 10, revealing that a deterministic volatility is enough to explain deterministic variations in temperature data, see e.g. [19]. Finally, we propose the following algorithm.

\footnotetext{
${ }^{4}$ In fact, we approximated the logarithmic average squared residuals by a truncated Fourier series as in (2) in order to ensure to obtain a positive estimate for $\psi_{1}(t)$.
} 


\section{Algorithm}

\section{Initialization}

Compute starting values $\hat{Z}_{2}(t)^{(0)}, \hat{\rho}^{(0)}, \hat{\lambda}^{(0)}$ based on DE; compute starting values $\hat{\sigma}_{2}^{(0)}$ based on an $\mathrm{AR}(1)$ model for $\hat{Z}_{2}(t)^{(0)}$; compute starting values $\hat{\gamma}^{(0)}$ for the fourier expansion coefficients based on NLSE.

\section{Iteration}

For $l=1,2, \ldots$ until convergence:

(a) Jointly maximize the likelihood (12) with respect to $\lambda, \rho, \sigma_{2}$ and the coefficients $\gamma$, corresponding to the Fourier expansion of $\psi(t)$, yielding ML-estimates $\hat{\lambda}^{(l)}, \hat{\rho}^{(l)}, \hat{\sigma}_{2}^{(l)}, \hat{\gamma}^{(l)}$.

(b) For each forecast curve at days $t=1, \ldots, 1740$, compute $\hat{Z}_{2}(t)^{(l)}$ as a weighted LS-estimate based on the de-seasonalized forecast curves in (5). Hence, for $t=$ $1, \ldots, 1740$, we have to solve:

$$
\underset{Z_{2}(t)}{\arg \min } \sum_{x=0}^{14}\left(\tilde{f}_{t ; t+x}-Z_{1}(t) e^{-\hat{\lambda}^{(l)} x}-\frac{Z_{2}(t)\left(e^{-\hat{\rho}^{(l)} x}-e^{-\hat{\lambda}^{(l)} x}\right)}{\hat{\lambda}^{(l)}-\hat{\rho}^{(l)}}\right)^{2} w_{x} .
$$

As this is a linear optimization problem, it can be solved analytically, yielding

$$
\hat{Z}_{2}(t)^{(l)}=\frac{\sum_{x=0}^{14}\left(\frac{e^{-\hat{\rho}^{(l)} x}-e^{-\hat{\lambda}^{(l)} x}}{\hat{\lambda}^{(l)}-\hat{\rho}^{(l)}}\right) w_{x}\left(\tilde{f}_{t ; t+x}-Z_{1}(t) e^{-\hat{\lambda}^{(l)} x}\right)}{\sum_{x=0}^{14}\left(\frac{e^{-\hat{\rho}^{(l)} x}-e^{-\hat{\lambda}^{(l)} x}}{\hat{\lambda}^{(l)}-\hat{\rho}^{(l)}}\right)^{2} w_{x}} .
$$

3. After convergence at step $l^{*}$, compute the final estimate $\hat{\psi}(t)$ based on $\hat{\gamma}^{\left(l^{*}\right)}$. Assuming a piece-wise constant process $\sigma_{1}(t)$ on the time intervals $[1,2), \ldots,[1739,1740)$, based on the estimate $\hat{\psi}(t)$ we can solve (11) with respect to $\sigma_{1}(t)$ and finally obtain an estimate $\hat{\sigma}_{1}(t)$.

For the non-linear maximization problem in step 2 (a) of the algorithm, we use the R-function bobyqa from the minqa-package, see [2], which provides an algorithm for bound constrained 


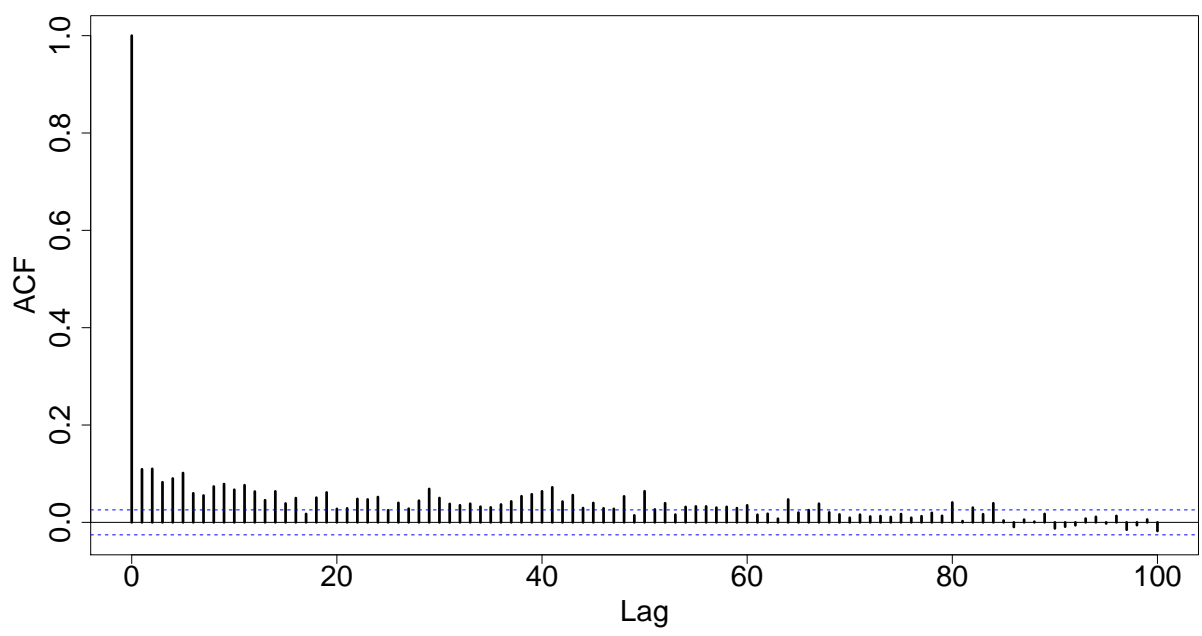

Figure 10: Autocorrelation function of the squared $A R(1)$-residuals on the New York data for the time period 1997-2012.

optimization without using derivatives. Note that, as temperature forecast are naturally less reliable the longer the forecast period lasts, we suggest to put more weight on shorter forecast periods and specify the following weight vector in step 2 (b) of the algorithm

$$
\mathbf{w}=\left(w_{0}, w_{1}, \ldots, w_{14}\right)^{\top}=(100, \ldots, 100,10, \ldots, 10,1, \ldots, 1)^{\top}
$$

with $w_{0}=\ldots=w_{4}=100, w_{5}=\ldots=w_{9}=10$ and $w_{10}=\ldots=w_{14}=1$. The final estimates for $\lambda, \rho$ and $\sigma_{2}$ yield $\hat{\lambda}^{\left(l^{*}\right)}=1.835, \hat{\rho}^{\left(l^{*}\right)}=0.107$ and $\hat{\sigma}_{2}^{\left(l^{*}\right)}=2.713$. The final fits of the forecast curves are illustrated in Figure 11, where we show the curve estimates together with the deseasonalized forecast data for 9 chosen days. We can see that the fitted curves do a rather good job within the bounds of possible curve features covered by the two-factor model (3), whereas on several days the fitted curves corresponding to a simple AR(1) model cannot reproduce the true forecast courses in a satisfactory way.

The final (annual) estimate for $\hat{\psi}(t)^{\left(l^{*}\right)}$, which is based on the final parameter estimates $\hat{\gamma}^{\left(l^{*}\right)}$, is plotted in Figure 12. It indicates that the standard deviation of the conditional distribution of the temperature in (11) has its maximum in February and its minimum at the end of July. This conforms with the findings in [10] and [19]. The corresponding piece-wise constant estimates for $\hat{\sigma}_{1}(t)$ yield values in the interval [2.584,3.351]. 
day 4

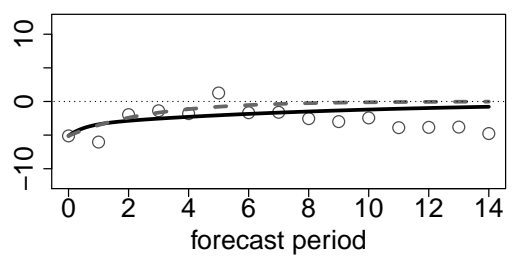

day 105

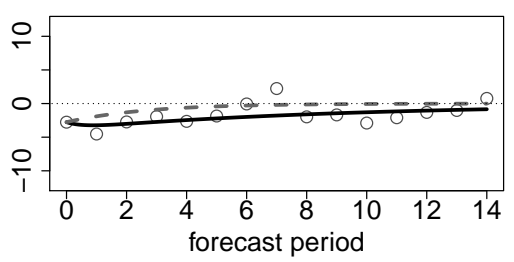

day 404

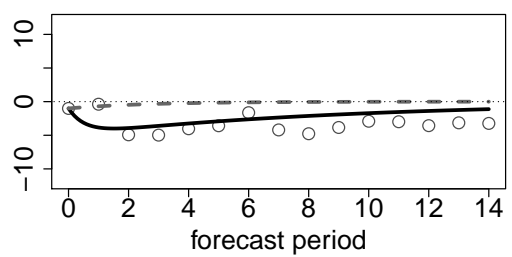

day 17

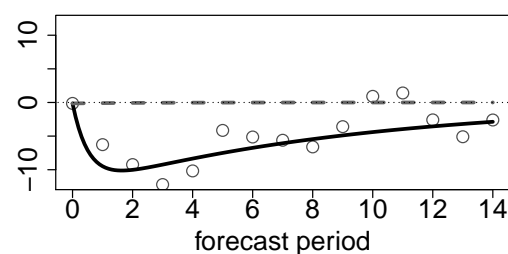

day 118

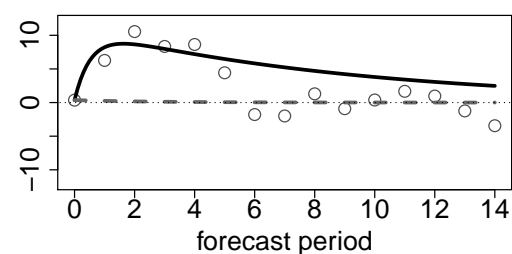

day 473

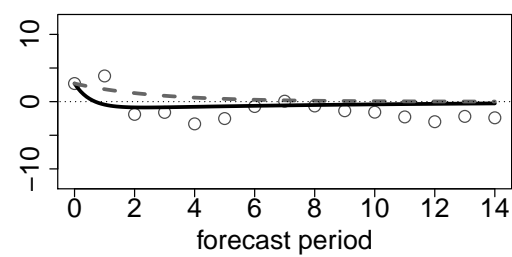

day 98

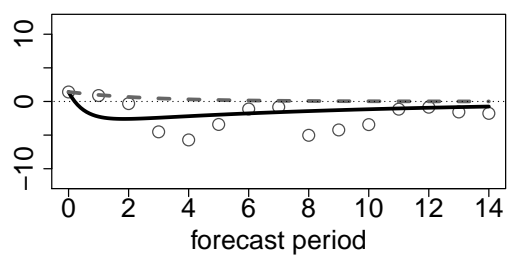

day 121

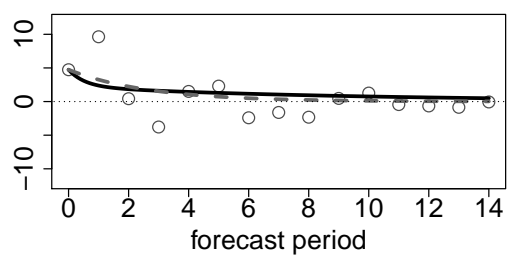

day 500

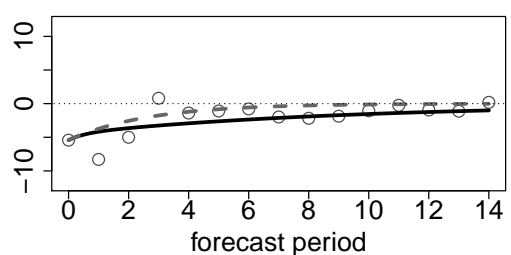

Figure 11: Estimated forecast curves for the two-factor model (black solid lines) and for a simple AR(1) model (gray dashed lines) together with de-seasonalized forecast data for 9 chosen days for New York.

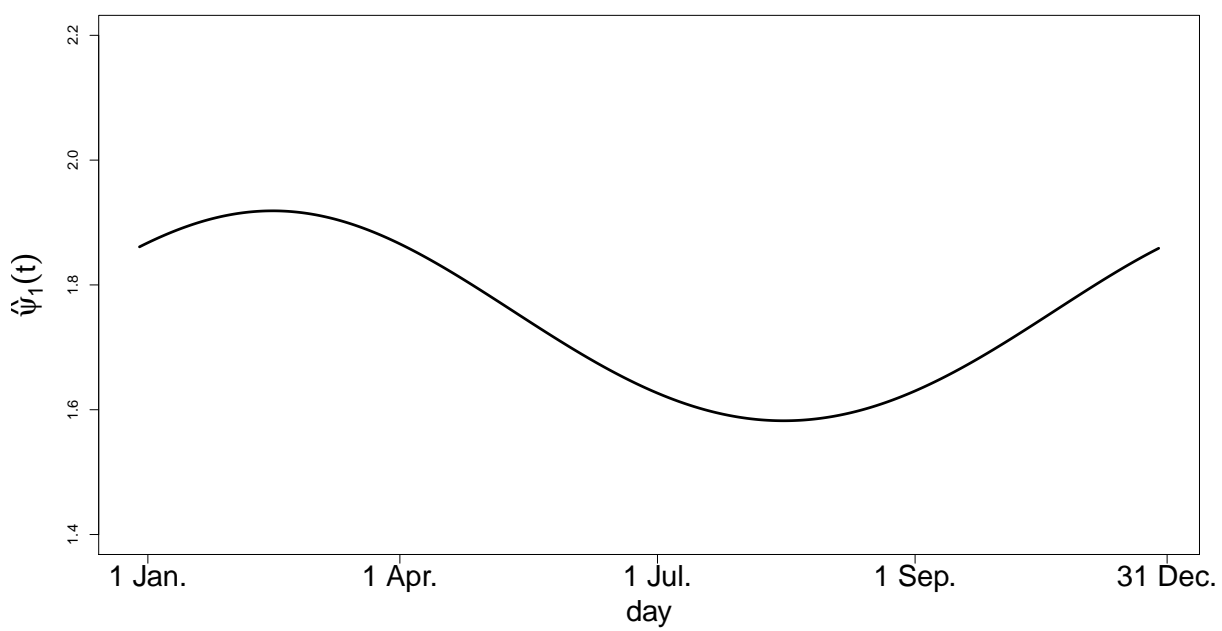

Figure 12: New York final (annual) estimate $\hat{\psi}(t)^{\left(l^{*}\right)}$, based on final parameter estimates $\hat{\gamma}^{\left(l^{*}\right)}$.

For Berlin, the final estimates for $\lambda, \rho$ and $\sigma_{2}$ yield $\hat{\lambda}^{\left(l^{*}\right)}=0.460, \hat{\rho}^{\left(l^{*}\right)}=0.120$ and $\hat{\sigma}_{2}^{\left(l^{*}\right)}=$ 0.962. The piece-wise constant estimates for $\hat{\sigma}_{1}(t)$ yield values in the interval [3.016,3.448]. Altogether, we obtain very similar results for Berlin and a comparable quality of the fitted curves with the major difference that the standard deviation of the conditional distribution of the temperature for Berlin has its maximum in September and its minimum at the beginning 
of April.

\section{Pricing Weather Derivatives}

Weather Derivatives started to trade at the Chicago Mercantile Exchange (CME) in the late '90s in order to hedge weather risk. Exchange-traded temperature derivatives are futures written on different temperature indices $I\left(T_{1}, T_{2}\right)$ measured over specified periods $\left[T_{1}, T_{2}\right]$ such as weeks, months or quarters of a year, and European options written on these futures. In the following we first derive explicit price formulas in our model for the most common futures contracts before we perform a calibration study on the market price of risk. The question of hedging and pricing options written on these futures is addressed in [21].

\subsection{CAT, HDD and CDD futures}

The most common temperature indices $I\left(T_{1}, T_{2}\right)$ are: Heating Degree Day (HDD), Cooling Degree Day (CDD), Cumulative Averages (CAT). The temperature indices take the accumulated average temperature over $\left[T_{1}, T_{2}\right]$ :

$$
\begin{aligned}
\operatorname{CAT}\left(T_{1}, T_{2}\right) & =\int_{T_{1}}^{T_{2}} \tau_{u} d u \\
\operatorname{CDD}\left(T_{1}, T_{2}\right) & =\int_{T_{1}}^{T_{2}} \max \left(\tau_{u}-C, 0\right) d u \\
\operatorname{HDD}\left(T_{1}, T_{2}\right) & =\int_{T_{1}}^{T_{2}} \max \left(C-\tau_{u}, 0\right) d u
\end{aligned}
$$

where $\tau_{u}$ denotes the daily average temperature. The measurement period is usually measured in standard months or seasons and $C$ is a threshold (typically $18^{\circ} \mathrm{C}$ or $65^{\circ} \mathrm{F}$ ) over a period $\left[T_{1}, T_{2}\right]$. HDD futures contracts are measured during November-April and for CDD and CAT futures contracts the measurement period is April-November.

According to no-arbitrage theory, pricing of financial assets with the temperature as underlying spot price has to be done under some risk-neutral pricing measure which in our setting can be any probability measure $\mathbb{Q}$ equivalent to $\mathbb{P}$ since the underlying cannot be traded. The futures price $F_{I}\left(t, T_{1}, T_{2}\right)$ written on a given temperature index $I\left(T_{1}, T_{2}\right)$ is chosen such that 
the value of the futures contract equals zero at emission time $t$ given the information $\mathcal{F}_{t}$, i.e. (assuming a deterministic risk-free rate for simplicity):

$$
\mathrm{E}_{Q}\left[I\left(T_{1}, T_{2}\right)-F_{I}\left(t, T_{1}, T_{2}\right) \mid \mathcal{F}_{t}\right]=0
$$

or

$$
F_{I}\left(t, T_{1}, T_{2}\right)=\mathrm{E}_{Q}\left[I\left(T_{1}, T_{2}\right) \mid \mathcal{F}_{t}\right]
$$

with $I\left(T_{1}, T_{2}\right)$ being one of the indices CAT, HDD or CDD.

We assume that the temperature dynamics of the temperature $f(t ; t)=\tau(t)$ under a riskneutral measure $\mathbb{Q}$ are given as $\Lambda(t)+Z_{1}(t)$, with

$$
d Z_{1}(t)=\left(\theta_{1}(t) \sigma_{1}(t)-\lambda Z_{1}(t)+Z_{2}(t)\right) d t+\sigma_{1}(t) d \widetilde{W}_{1}(t)
$$

and

$$
d Z_{2}(t)=\left(\theta_{2}(t) \sigma_{2}-\rho Z_{2}(t)\right) d t+\sigma_{2}(t) d \widetilde{W}_{2}(t)
$$

where $d \widetilde{W}_{1}(t):=d W_{1}(t)-\theta_{1}(t) d t$ and $d \widetilde{W}_{2}(t):=d W_{2}(t)-\theta_{2}(t) d t$ define independent $\mathbb{Q}$ Brownian motions and the market price of risk $\theta=\left(\theta_{1}, \theta_{2}\right)$ consists of some bounded deterministic functions. This imposes a certain restriction on the set of possible pricing measures, but simplifies the calculations considerably. We first consider the pricing of CAT futures.

Proposition 1. For $t \leq T_{1}$, the CAT futures price $F^{C A T}\left(t ; T_{1}, T_{2}\right)$ is given by

$$
\begin{aligned}
F^{C A T}\left(t ; T_{1}, T_{2}\right) & =\int_{T_{1}}^{T_{2}} \Lambda(s) d s-Z_{1}(t) l_{T_{1}, T_{2}}^{\lambda}(t)+Z_{2}(t) \frac{l_{T_{1}, T_{2}}^{\rho}(t)-l_{T_{1}, T_{2}}^{\lambda}(t)}{\rho-\lambda} \\
& -\int_{t}^{T_{2}} \theta_{1}(u) \sigma_{1}(u) l_{u \vee T_{1}, T_{2}}^{\lambda}(u) d u \\
& +\int_{t}^{T_{2}} \theta_{2}(u) \sigma_{2} \frac{l_{u \vee T_{1}, T_{2}}^{\rho}(u)-l_{u \vee T_{1}, T_{2}}^{\lambda}(u)}{\rho-\lambda} d u
\end{aligned}
$$


where the function $l_{R, S}^{\alpha}(x)$ is defined by

$$
l_{R, S}^{\alpha}(x):=\frac{e^{-\alpha(S-x)}-e^{-\alpha(R-x)}}{\alpha} ; \quad \alpha, R, S, x \in \mathbb{R}
$$

and $x \vee y:=\max \{x, y\}$. For $T_{1}<t \leq T_{2}$ we obtain

$$
F^{C A T}\left(t ; T_{1}, T_{2}\right)=\int_{T_{1}}^{t} \tau(s) d s+F^{C A T}\left(t ; t, T_{2}\right)
$$

In particular, for the special case $\theta_{1}(u)=\theta_{1}, \theta_{2}(u)=\theta_{2}, \sigma_{1}(u)=\sigma_{1}$ constant on $\left[t, T_{2}\right]$, Proposition 1 gives by direct computation the following CAT futures price for $t \leq T_{1}$ :

$$
\begin{aligned}
F^{\mathrm{CAT}}\left(t ; T_{1}, T_{2}\right) & =\int_{T_{1}}^{T_{2}} \Lambda(s) d s-l_{T_{1}, T_{2}}^{\lambda}(t)\left(Z_{1}(t)+\frac{Z_{2}(t)}{\rho-\lambda}-\frac{\theta_{1} \sigma_{1}}{\lambda}-\frac{\theta_{2} \sigma_{2}}{\lambda(\rho-\lambda)}\right) \\
& +l_{T_{1}, T_{2}}^{\rho}(t)\left(\frac{Z_{2}(t)}{\rho-\lambda}-\frac{\theta_{2} \sigma_{2}}{\rho(\rho-\lambda)}\right)+\left(T_{2}-T_{1}\right)\left(\frac{\theta_{1} \sigma_{1}}{\lambda}+\frac{\theta_{2} \sigma_{2}}{\rho \lambda}\right) .
\end{aligned}
$$

Proof. From (13) we obtain with $I\left(T_{1}, T_{2}\right)$ the CAT index

$$
F^{\mathrm{CAT}}\left(t ; T_{1}, T_{2}\right)=\mathrm{E}_{\mathbb{Q}}\left[\int_{T_{1}}^{T_{2}} \tau(s) d s \mid \mathcal{F}_{t}\right] .
$$

By Fubini's theorem, we can rewrite

$$
F^{\mathrm{CAT}}\left(t ; T_{1}, T_{2}\right)=\int_{T_{1}}^{T_{2}} f_{\mathbb{Q}}(t ; s) d s
$$

with

$$
f_{\mathbb{Q}}(t ; s):=\mathrm{E}_{\mathbb{Q}}\left[\tau(s) \mid \mathcal{F}_{t}\right]=\Lambda(s)+\mathrm{E}_{\mathbb{Q}}\left[Z_{1}(s) \mid \mathcal{F}_{t}\right]
$$

Now recall from (9) and (10) that for $s \geq t$

$$
\begin{aligned}
Z_{1}(s) & =Z_{1}(t) e^{-\lambda(s-t)}+\int_{t}^{s} \sigma_{1}(u) e^{-\lambda(s-u)} d W_{1}(u) \\
& +Z_{2}(t) \frac{\left(e^{-\rho(s-t)}-e^{-\lambda(s-t)}\right)}{\lambda-\rho}+\int_{t}^{s} \int_{r}^{s} \sigma_{2}(r) e^{-\lambda(s-u)-\rho(u-r)} d u d W_{2}(r)
\end{aligned}
$$

Rewriting (14) in terms of $\widetilde{W}_{1}$ and $\widetilde{W}_{2}$ and taking conditional expectation with respect to $\mathbb{Q}$ 
gives

$$
\begin{aligned}
f_{\mathbb{Q}}(t ; s) & =\Lambda(s)+Z_{1}(t) e^{-\lambda(s-t)}+Z_{2}(t) \frac{e^{-\rho(s-t)}-e^{-\lambda(s-t)}}{\lambda-\rho} \\
& +\int_{t}^{s} \theta_{1}(u) \sigma_{1}(u) e^{-\lambda(s-u)} d u+\int_{t}^{s} \theta_{2}(u) \sigma_{2}(u) \frac{e^{-\rho(s-u)}-e^{-\lambda(s-u)}}{\lambda-\rho} d u .
\end{aligned}
$$

Finally, using Fubini again, we can compute the CAT-futures price for $t \leq T_{1}$ as

$$
\begin{aligned}
F^{\mathrm{CAT}}\left(t ; T_{1}, T_{2}\right) & =\int_{T_{1}}^{T_{2}} f_{\mathbb{Q}}(t ; s) d s \\
& =\int_{T_{1}}^{T_{2}} \Lambda(s) d s-Z_{1}(t) l_{T_{1}, T_{2}}^{\lambda}(t)+Z_{2}(t) \frac{l_{T_{1}, T_{2}}^{\rho}(t)-l_{T_{1}, T_{2}}^{\lambda}(t)}{\rho-\lambda} \\
& -\int_{t}^{T_{2}} \theta_{1}(u) \sigma_{1}(u) l_{u \vee T_{1}, T_{2}}^{\lambda}(u) d u \\
& +\int_{t}^{T_{2}} \theta_{2}(u) \sigma_{2}(u) \frac{l_{u \vee T_{1}, T_{2}}^{\rho}(u)-l_{u \vee T_{1}, T_{2}}^{\lambda}(u)}{\rho-\lambda} d u
\end{aligned}
$$

For $T_{1}<t \leq T_{2}$ we obtain

$$
\begin{aligned}
F^{\mathrm{CAT}}\left(t ; T_{1}, T_{2}\right) & =\int_{T_{1}}^{t} \tau(s) d s+\mathrm{E}_{\mathbb{Q}}\left[\int_{t}^{T_{2}} \tau(s) d s \mid \mathcal{F}_{t}\right] \\
& =\int_{T_{1}}^{t} \tau(s) d s+F^{\mathrm{CAT}}\left(t ; t, T_{2}\right) .
\end{aligned}
$$

Next, we turn our attention to HDD and CDD futures:

Proposition 2. For $t \leq T_{1}$, the HDD futures price is given by

$$
\begin{aligned}
F^{H D D}\left(t ; T_{1}, T_{2}\right) & =\int_{T_{1}}^{T_{2}}\left\{\left(C-\Lambda(s)-\tilde{\mu}_{t, s}\right) \Phi\left(\frac{C-\Lambda(s)-\tilde{\mu}_{t, s}}{\tilde{\psi}_{t, s}}\right)\right. \\
& \left.+\tilde{\psi}_{t, s} \phi\left(\frac{C-\Lambda(s)-\tilde{\mu}_{t, s}}{\tilde{\psi}_{t, s}}\right)\right\} d s,
\end{aligned}
$$


and the CDD futures price is given by

$$
\begin{aligned}
F^{C D D}\left(t ; T_{1}, T_{2}\right) & =\int_{T_{1}}^{T_{2}}\left\{\left(\Lambda(s)+\tilde{\mu}_{t, s}-C\right) \Phi\left(\frac{\Lambda(s)+\tilde{\mu}_{t, s}-C}{\tilde{\psi}_{t, s}}\right)\right. \\
& \left.+\tilde{\psi}_{t, s} \phi\left(\frac{\Lambda(s)+\tilde{\mu}_{t, s}-C}{\tilde{\psi}_{t, s}}\right)\right\} d s,
\end{aligned}
$$

where $\Phi$ is the cumulative distribution function, $\phi$ the density of the standard normal distribution, and

$$
\begin{aligned}
\tilde{\mu}_{t, s} & =Z_{1}(t) e^{-\lambda(s-t)}+Z_{2}(t) \frac{e^{-\rho(s-t)}-e^{-\lambda(s-t)}}{\lambda-\rho} \\
& +\int_{t}^{s} \theta_{1}(u) \sigma_{1}(u) e^{-\lambda(s-u)} d u+\int_{t}^{s} \theta_{2}(u) \sigma_{2} \frac{e^{-\rho(s-u)}-e^{-\lambda(s-u)}}{\lambda-\rho} d u \\
\tilde{\psi}_{t, s}^{2} & =\int_{t}^{s} \sigma_{1}^{2}(u) e^{-2 \lambda(s-u)} d u+\int_{t}^{s}\left(\int_{r}^{s} \sigma_{2} e^{-\lambda(s-u)-\rho(u-r)} d u\right)^{2} d r .
\end{aligned}
$$

For $T_{1}<t \leq T_{2}$ we obtain

$$
\begin{aligned}
& F^{H D D}\left(t ; T_{1}, T_{2}\right)=\int_{T_{1}}^{t}(C-\tau(s))^{+} d s+F^{H D D}\left(t ; t, T_{2}\right), \\
& F^{C D D}\left(t ; T_{1}, T_{2}\right)=\int_{T_{1}}^{t}(\tau(s)-C)^{+} d s+F^{C D D}\left(t ; t, T_{2}\right) .
\end{aligned}
$$

Proof. The HDD futures price is given by

$$
\begin{aligned}
F^{\mathrm{HDD}}\left(t ; T_{1}, T_{2}\right) & =\mathrm{E}_{\mathbb{Q}}\left[\int_{T_{1}}^{T_{2}}(C-\tau(s))^{+} d s \mid \mathcal{F}_{t}\right] \\
& =\int_{T_{1}}^{T_{2}} \mathrm{E}_{\mathbb{Q}}\left[(C-\tau(s))^{+} \mid \mathcal{F}_{t}\right] d s .
\end{aligned}
$$

Now, for a Gaussian random variable $X \sim \mathrm{N}\left(\mu ; \sigma^{2}\right)$ straight forward calculations give

$$
\mathrm{E}_{\mathbb{Q}}\left[(C-X)^{+}\right]=(C-\mu) \Phi\left(\frac{C-\mu}{\sigma}\right)+\sigma \phi\left(\frac{C-\mu}{\sigma}\right)
$$


where $C$ is a constant and $\Phi$ and $\phi$ are as in Proposition 2 above.

Rewriting (14) in terms of $\widetilde{W}_{1}$ and $\widetilde{W}_{2}$ one sees that the conditional distribution of $Z_{1}(s)$ given $Z(t)$ for $s \geq t \geq 0$ under $\mathbb{Q}$ is

$$
Z_{1}(s) \mid Z(t) \sim \mathrm{N}\left(\tilde{\mu}_{t, s} ; \tilde{\psi}_{t, s}^{2}\right)
$$

where $\tilde{\mu}_{t, s}$ and $\tilde{\psi}_{t, s}^{2}$ are given in Proposition 2 above. Since $\tau(s)=\Lambda(s)+Z_{1}(s)$ the result follows.

For CDD futures prices the computations are analogue.

\subsection{Calibration of the market price of risk}

We now turn our attention to the calibration of the market price of risk, i.e. to the calibration of the pricing measure used by the market to price temperature derivatives, implied by our model. In a previous study of temperature markets by [19] it was found that the calibrated market price of risk behaves very irregular in time, in particular when times to futures' maturities become short. In that study, the temperature is modeled by a CAR(3) model (continuous time autoregressive of order 3) and the information available to the market is modeled by the filtration generated by the temperature.

However, as argued in the introduction, we believe that at least parts of the irregular behavior of the market price of risk in [19] is not due to irregular risk pricing of the market but due to a misspecification of the model that is used to calibrate risk prices: forward looking information about the temperature available to the market is not taken into account in the information modeling. Obviously, when the information available to the market is assumed to be generated only by the past temperature, substantial amounts of forward looking information available to the market is not taken care of in [9] and [19].

In our approach we include, at least essential parts of, available forward looking information by specifying a model for the complete forecast curve and the intention of this section is now to estimate the market price of risk structure implied by our model approach and to compare it to 
the market price of risk obtained without meteorological forecast information. To this end, we calibrate model implied futures prices to the market and proceed as follows. For simplicity, we assume the market prices of risk $\theta_{1}(u)=\theta_{1}$ and $\theta_{2}(u)=\theta_{2}$ to be constant. Then Proposition 2 yields by direct computations:

$$
\begin{aligned}
\tilde{\mu}_{t, s} & =Z_{1}(t) e^{-\lambda(s-t)}+Z_{2}(t) \frac{e^{-\rho(s-t)}-e^{-\lambda(s-t)}}{\lambda-\rho} \\
& +\theta_{1} \int_{t}^{s} \sigma_{1}(u) e^{-\lambda(s-u)} d u+\frac{\theta_{2} \sigma_{2}}{\lambda-\rho}\left\{\frac{1-e^{-\rho(s-t)}}{\rho}+\frac{1-e^{-\lambda(s-t)}}{\lambda}\right\} .
\end{aligned}
$$

and

$$
\begin{aligned}
\tilde{\psi}_{t, s}^{2} & =\int_{t}^{s} \sigma_{1}^{2}(u) e^{-2 \lambda(s-u)} d u \\
& +\frac{\sigma_{2}^{2}}{(\lambda-\rho)^{2}}\left\{\frac{1-e^{-2 \rho(s-t)}}{2 \rho}+\frac{1-e^{-2 \lambda(s-t)}}{2 \lambda}-\frac{2\left(1-e^{-(\rho+\lambda)(s-t)}\right)}{\rho+\lambda}\right\} .
\end{aligned}
$$

We got access to prices for 7 CAT futures contracts for the region of Berlin-Tempelhof airport from the Chicago Mercantile Exchange (CME) traded within the time period 2008122920091031 and measurement period (April - Nov) as well as prices of 45 HDD and CDD futures contracts for the region of New York-JFK airport ${ }^{5}$ within the time period 20081229-20120330. In the following we use the formulas from Proposition 2 to compute the course of the market price of risk, based on the model parameter estimates derived in Section 3.2. For comparison, we also compute the market prices of risk based on a simple $\mathrm{AR}(1)$ model, which does not include any meteorological forecast information and is solely estimated on past temperatures. Note here that as the European CAT futures market is hardly liquid and thus the CAT futures price time series for Berlin are mostly constant, even within the delivery period, also the corresponding courses of the market price of risk $\theta_{1}$ are basically constant lines, with some spikes close to delivery. Hence, we restrict our analysis in the following on the New York data, in particular on New York HDD futures prices.

For both methods we successively derive the market price of risk $\hat{\theta}_{1}(t)$ for all days $t$ where futures prices of the considered HDD were available to us, by solving the equality for the HDD

\footnotetext{
${ }^{5}$ The temperature futures were obtained from Bloomberg.
} 
futures price $F^{\mathrm{HDD}}\left(t ; T_{1}, T_{2}\right)$ from Proposition 2 with respect to $\theta_{1}$. For the $\mathrm{AR}(1)$ approach we simply set both $Z_{2}(t)$ and $\sigma_{2}^{2}$ equal to zero.

At this point we want to recall the data inconsistencies of temperature and forecasts already mentioned in Footnote 1 and in Section 2.1 which are a possible error source concerning the results of our market price of risk calibrations. These inconsistencies don't allow to fully exploit the forward looking information contained in meteorological forecasts. So the performance of our model parameter estimates and hence, the quality of our market price of risk calibrations could potentially be considerably improved, if the forecast data would correspond to the temperature data.

In Figure 13 and Figure 14 we show the results for a selection of New York HDD futures based on our two-factor model from (3) (black solid line) and based on the AR(1) approach (gray dashed line). We find that in all investigated scenarios the courses of the market prices of risk are generally similar for both used methods and at a first glance no major differences are visible. To make the courses comparable we have standardized them, always taking the first available day of the futures price time series as the reference point. For both methods, the market price of risk is quite steady before the delivery period begins, but then becomes more and more turbulent when getting close to delivery.

But if we have a closer look on the total relative variation in the courses, corresponding to a given series of HDD futures prices on a time interval $\left[t, T_{2}\right]$, i.e.

$$
T V:=\sum_{s=t}^{T_{2}-1} \frac{\left|\hat{\theta}_{1}(s+1)-\hat{\theta}_{1}(s)\right|}{\left|\hat{\theta}_{1}(s)\right|}
$$

we find that the market prices of risk based on our two-factor model behave more regular than those based on the $\mathrm{AR}(1)$ approach, both on the whole time interval and during the delivery period, compare Table 2 and Table 3. Though the differences might not seem to be crucial, we still can observe the trend that a model accounting for forward looking information leads to more regular market prices of risk. In particular, the quality of the incorporated forward looking information in the two-factor model could be probably considerably improved, if the data inconsistencies mentioned above could be eliminated. 
MPR for HDD No. 1

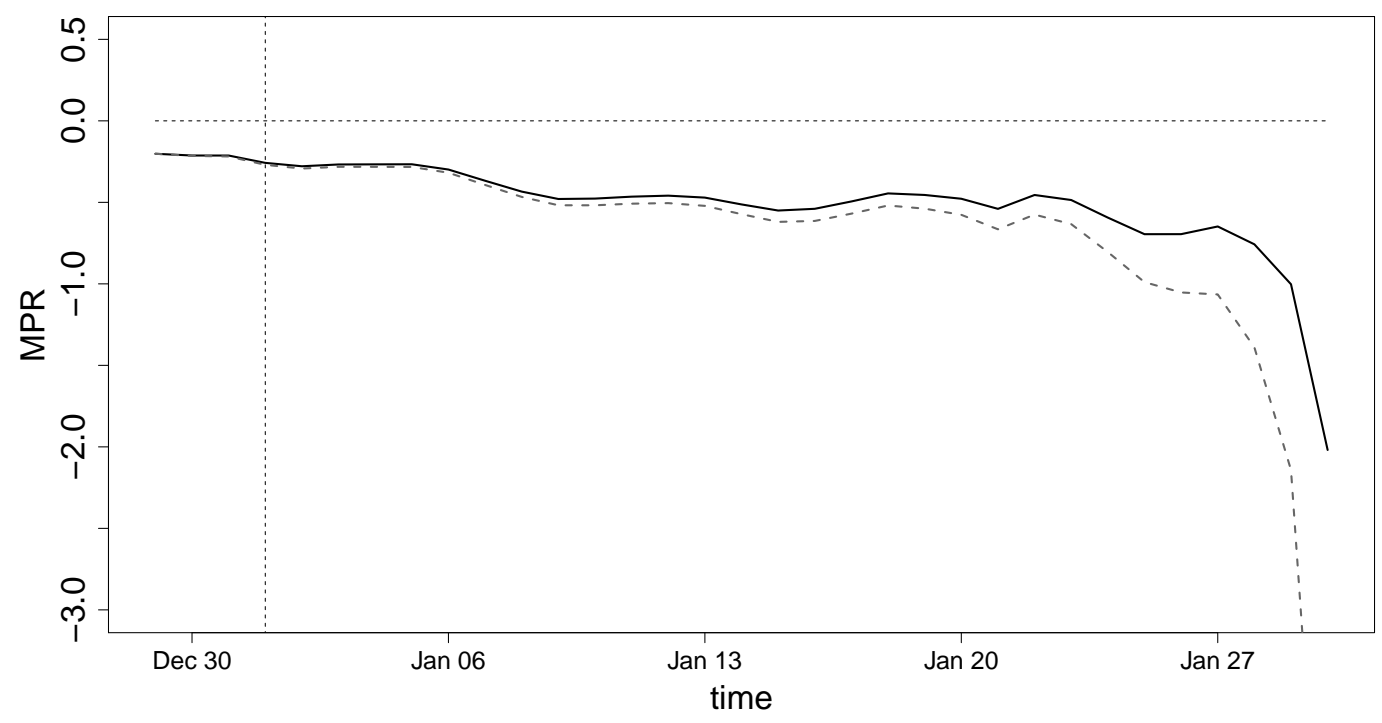

MPR for HDD No. 14

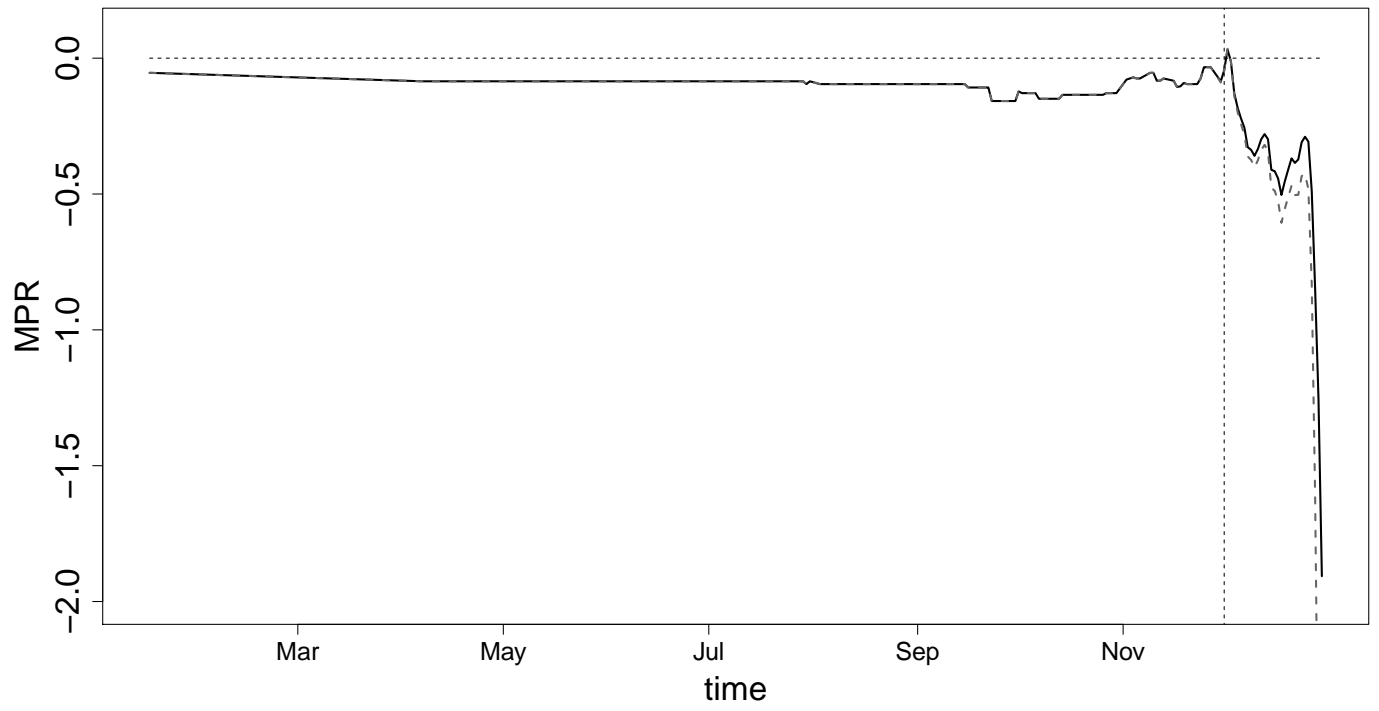

Figure 13: Standardized market price of risk courses for a selection of New York HDD futures prices based on the two-factor model from (3) (black solid line) and on an AR(1) model (gray dashed line); the start of the delivery period is indicated by the dashed vertical line. 


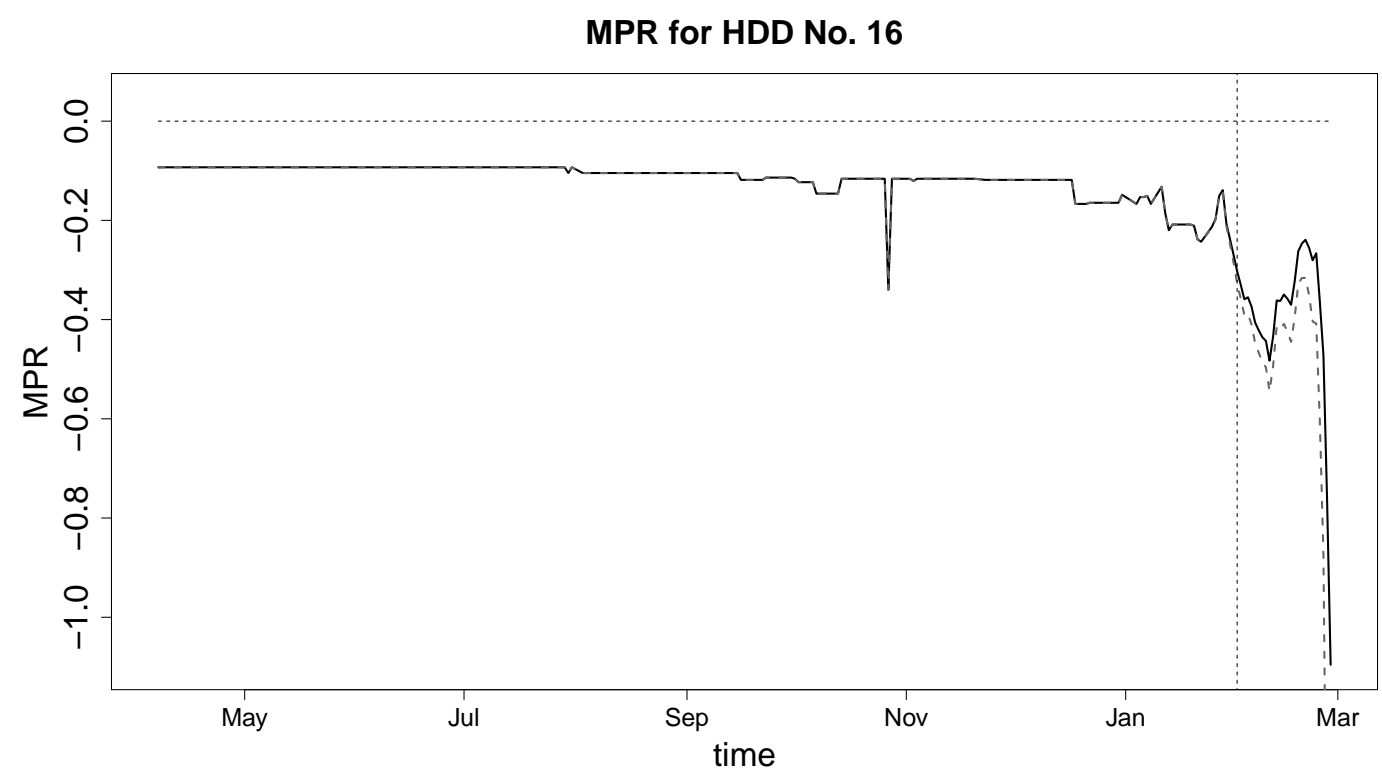

MPR for HDD No. 29

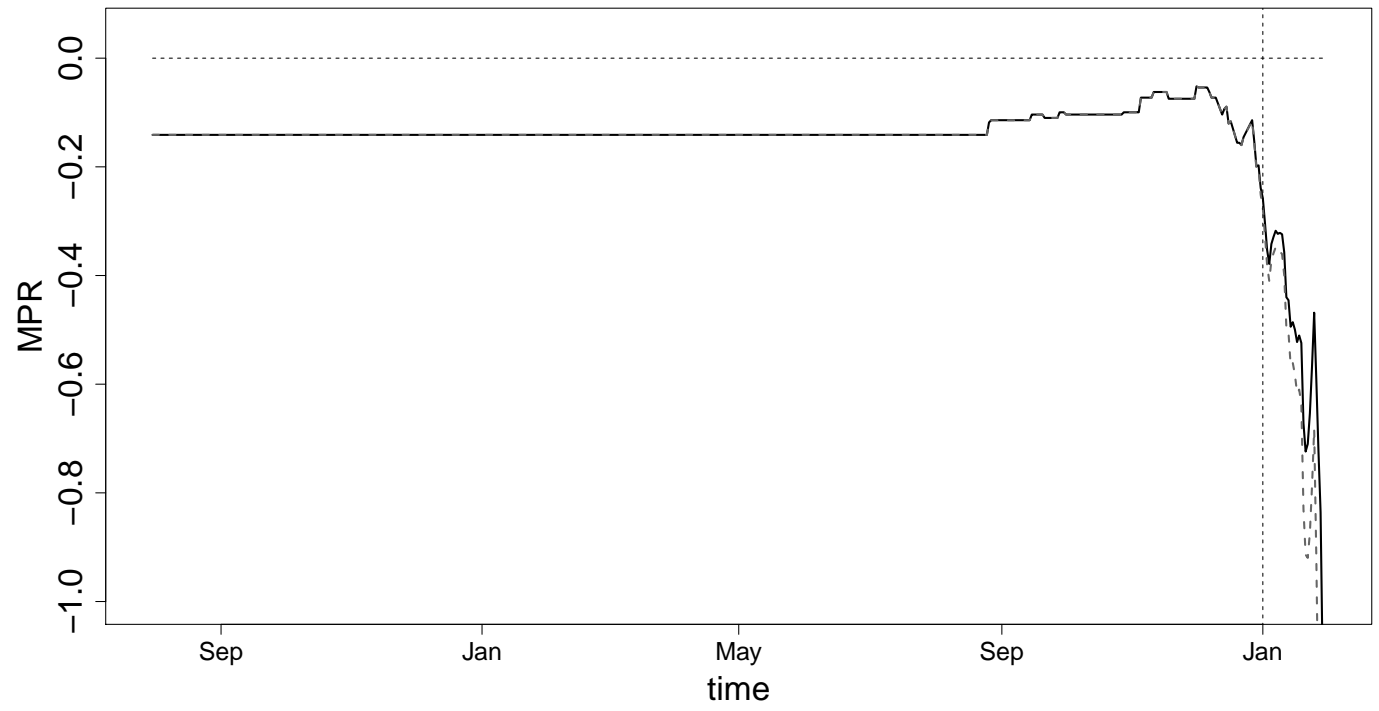

Figure 14: Standardized market price of risk courses for a selection of New York HDD futures prices based on the two-factor model from (3) (black solid line) and on an AR(1) model (gray dashed line); the start of the delivery period is indicated by the dashed vertical line. 


\begin{tabular}{lcccc}
\hline HDD No. & 1 & 14 & 16 & 29 \\
\hline$T V_{\mathrm{AR}(1)}$ & 4.93 & 25.60 & 11.13 & 9.26 \\
$T V_{2 \text {-factor }}$ & 3.86 & 24.51 & 10.17 & 8.22 \\
\hline
\end{tabular}

Table 2: Difference in total relative variation between the two-factor and AR(1) model for a selection of New York HDD futures.

\begin{tabular}{lcccc}
\hline HDD No. & 1 & 14 & 16 & 29 \\
\hline$T V_{\mathrm{AR}(1)}$ & 4.62 & 19.07 & 4.11 & 4.91 \\
$T V_{2 \text {-factor }}$ & 3.59 & 18.02 & 3.21 & 3.91 \\
\hline
\end{tabular}

Table 3: Difference in total relative variation between the two-factor and AR(1) model for a selection of New York HDD futures (only in the delivery period).

\section{Conclusion}

We propose a consistent two-factor model for pricing temperature derivatives that incorporates the forward looking information available in the market by specifying a reduced model for the complete temperature forecast curve in addition to the evolution of the temperature. The two factors describing the tilting or bending of the curves reflect accurately the stylized facts of temperature and temperature forecast in the short end. The two-factor model allows for factors with mean-reversion to a stochastic mean level. We find that the market prices of risk, calibrated from CME temperature derivatives, based on our two-factor models behave more regular than those based on an $\mathrm{AR}(1)$-Ornstein-Uhlenbeck approach, both in and out of the measurement period. 


\section{References}

[1] Alaton, P., Djehiche, B., and Stillberger, D. (2002). On modelling and pricing weather derivatives. Appl. Math. Finance, 9(1):1-20.

[2] Bates, D., Mullen, K. M., Nash, J. C., and Varadhan, R. (2012). minqa: Derivative-free optimization algorithms by quadratic approximation. R package version 1.2.1.

[3] Bates, D. M. and Watts, D. G. (1988). Nonlinear Regression Analysis and Its Applications. Wiley.

[4] Bellman, R. E. (1961). Adaptive Control Processes: A Guided Tour. Princeton University Press, Princeton.

[5] Benth, F., Biegler-König, R., and Kiesel, R. (2013). An empirical study of the information premium on electricity markets. Energy Economics, 36:55-77.

[6] Benth, F. and Meyer-Brandis, T. (2009). The information premium for non-storable commodities. Journal of Energy Market, 2.

[7] Benth, F. E. and Benth, S. (2011). Weather derivatives and stochastic modelling of temperature. International Journal of Stochastic Analysis, 2011:1-21.

[8] Benth, F. E., Cartea, A., and Kiesel, R. (2008). Pricing forward contracts in power markets by the certainty equivalence principle: Explaining the sign of the market risk premium. Journal of Banking and Finance, 32(10):2006-2021.

[9] Benth, F. E. and Saltyte Benth, J. (2012). Modeling and Pricing in Financial Markets for Weather Derivatives. Band 17, Advanced Series on Statistical Science and Applied Probability, World Scientific Publishing Company Incorporated.

[10] Benth, F. E., Saltyte Benth, J., and Koekebakker, S. (2007). Putting a price on temperature. Scandinavian Journal of Statistics, 12(1):53-85.

[11] Brockett, P., Wang, M., Yang, C., and Zou, H. (2006). Portfolio effects and valuation of weather derivatives. Financial Review, 41(1):55-76. 
[12] Campbell, S. and Diebold, F. (2005). Weather forecasting for weather derivatives. Journal of American Statistical Association, 100(469):6-16.

[13] Cao, M. and Wei, J. (2004). Weather derivatives valuation and market price of weather risk. The Journal of Future Markets, 24(11):1065-1089.

[14] Dorfleitner, G. and Wimmer, M. (2010). The pricing of temperature futures at the chicago mercantile exchange. Journal of Banking and Finance, 34(6):1360-1370.

[15] Filipovic, D. (1999). A note on the nelson-siegel family. Mathematical Finance, 9:349-359.

[16] Gilli, M., Große, S., and Schumann, E. (2010). Calibrating the Nelson-Siegel-Svensson model. COMISEF Working Paper Series No. 31. available from http://comisef.eu/?q= working_papers.

[17] Gilli, M., Maringer, D., and Schumann, E. (2011). Numerical Methods and Optimization in Finance. Academic Press.

[18] Härdle, W., López-Cabrera, B., and Ritter, M. (2012). Forecast based pricing of weather derivatives. SFB 649 Discussion Paper 2012-02\%.

[19] Härdle, W. K. and López-Cabrera, B. (2012). The implied market price of weather risk. Applied Mathematical Finance, 19(1):59-95.

[20] Härdle, W. K., López-Cabrera, B., Okhrin, O., and Wang, W. (2011). Localizing temperature risk. SFB 649 Discussion Paper 2011-01, Humboldt-Universität zu Berlin. 2nd Revision in Journal of Econometrics.

[21] Hell, P., Meyer-Brandis, T., and Rheinländer, T. (2012). Consistent factor models for temperature markets. International Journal of Theoretical and Applied Finance, 15(4):1250027$1-1250027-24$.

[22] Huang-Hsi, H., Yung-Ming, S., and Pei-Syun, L. (2008). Hdd and cdd option pricing with market price of weather risk for taiwan. The Journal of Future Markets, 28(8):790-814. 
[23] Jewson, S. and Brix, A. (2005). Weather Derivative Valuation. Cambridge University Press, Cambridge.

[24] Nelson, C. R. and Siegel, A. F. (1987). Parsimonious modeling of yield curves. The Journal of Business, 60(4):473-489.

[25] R Core Team (2013). R: A Language and Environment for Statistical Computing. R Foundation for Statistical Computing, Vienna, Austria.

[26] Ramsay, J. O. and Silverman, B. W. (2005). Functional Data Analysis. Springer, New York, 2nd edition.

[27] Richards, T., Manfredo, M., and Sanders, D. (2004). Pricing weather derivatives. American Journal of Agricultural Economics, 86(4):1005-1017.

[28] Ritter, M., Musshoff, O., and Odening, M. (2011). Meteorological forecasts and the pricing of temperature futures. Journal of Derivatives, 19.

[29] Shang, H. L. (2013). A survey of functional principal component analysis. Advances in Statistical Analysis.

[30] Viviani, R., Grön, G., and Spitzer, M. (2005). Functional principal component analysis of fMRI data. Human Brain Mapping, 24:109-129.

[31] Yoo, S. (2004). Weather derivatives and seasonal forecast. Asia-Pacific Journal of Financial Studies, pages 213-246. 


\section{SFB 649 Discussion Paper Series 2014}

For a complete list of Discussion Papers published by the SFB 649, please visit http://sfb649.wiwi.hu-berlin.de.

001 "Principal Component Analysis in an Asymmetric Norm" by Ngoc Mai Tran, Maria Osipenko and Wolfgang Karl Härdle, January 2014.

002 "A Simultaneous Confidence Corridor for Varying Coefficient Regression with Sparse Functional Data" by Lijie Gu, Li Wang, Wolfgang Karl Härdle and Lijian Yang, January 2014.

003 "An Extended Single Index Model with Missing Response at Random" by Qihua Wang, Tao Zhang, Wolfgang Karl Härdle, January 2014.

004 "Structural Vector Autoregressive Analysis in a Data Rich Environment: A Survey" by Helmut Lütkepohl, January 2014.

005 "Functional stable limit theorems for efficient spectral covolatility estimators" by Randolf Altmeyer and Markus Bibinger, January 2014.

006 "A consistent two-factor model for pricing temperature derivatives" by Andreas Groll, Brenda López-Cabrera and Thilo Meyer-Brandis, January 2014 